\title{
Plant and Human MORC Proteins Have DNA-Modifying Activities Similar to Type II Topoisomerases, but Require One or More Additional Factors for Full Activity
}

\author{
Murli Manohar, ${ }^{1}$ Hyong Woo Choi, ${ }^{1}$ Patricia Manosalva, ${ }^{1}$ Caroline A. Austin, ${ }^{2}$ Joseph E. Peters,${ }^{3}$ and \\ Daniel F. Klessig ${ }^{1}$
}

${ }^{1}$ Boyce Thompson Institute, Ithaca, NY 14853, U.S.A.; ${ }^{2}$ Institute for Cell and Molecular Biosciences, Newcastle University, NE2 $4 \mathrm{HH}$, U.K.; and ${ }^{3}$ Department of Microbiology, Cornell University, Ithaca, NY 14853, U.S.A.

Accepted 16 December 2016.

\begin{abstract}
To elucidate one or more mechanisms through which microrchidia (MORC) proteins impact immunity, epigenetic gene silencing, and DNA modifications, the enzymatic activities of plant MORCs were characterized. Previously, we showed that plant MORC1s have ATPase and DNA endonuclease activities. Here, we demonstrate that plant MORCs have topoisomerase type II (topo II)-like activities, as they i) covalently bind DNA, ii) exhibit DNA-stimulated ATPase activity, iii) relax or nick supercoiled DNA, iv) catenate DNA, and v) decatenante kinetoplast DNA. Mutational analysis of tomato SIMORC1 suggests that a $\mathrm{K}$ loop-like sequence is required to couple DNA binding to ATPase stimulation as well as for efficient SIMORC1's DNA relaxation and catenation activities and in planta suppression of INF1-induced cell death, which is related to immunity. Human MORCs were found to exhibit the same topo IIlike DNA modification activities as their plant counterparts. In contrast to typical topo IIs, SIMORC1 appears to require one or more accessory factors to complete some of its enzymatic activities, since addition of tomato extracts were needed for ATP-dependent, efficient conversion of supercoiled DNA to nicked/relaxed DNA and catenanes and for formation of topoisomer intermediates. Both plant and human MORCs bind salicylic acid; this suppresses their decatenation but not relaxation activity.
\end{abstract}

Microrchidia (MORC) proteins belong to a rather poorly characterized family of proteins that have been identified in prokaryotes and eukaryotes. They are defined by two hallmark domains, a GHKL-type ATPase and an S5 fold (Iyer et al. 2008). Humans have five MORC family members, while the model plant species Arabidopsis thaliana contains seven. Emerging data suggest their participation in signal-dependent chromatin remodeling and epigenetic regulation ( $\mathrm{Li}$ et al. 2013). In addition, all five human MORCs (HsMORCs) have been associated with cancer. Elevated expression of or

Current address for P. Manosalva: Department of Plant Pathology and Microbiology, University of California Riverside, Riverside, CA 92521, U.S.A.

Corresponding authors: D. F. Klessig and J. E. Peters;

E-mails: dfk8@cornell.edu and jep48@cornell.edu;

Telephone: +1.607.254.4560 and +1.607.255.2271.

*The $\boldsymbol{e}$-Xtra logo stands for "electronic extra" and indicates that seven supplementary figures are published online.

@ 2017 The American Phytopathological Society mutations in HsMORC1, HsMORC2, and HsMORC4 are linked to breast cancer, multiple myeloma, carcinomas, or B cell lymphoma, while HsMORC3 and SMCHD1(structural maintenance of chromosomes hinge domain containing 1), which corresponds to the fifth member of human MORC proteins, are associated with tumor suppression (Li et al. 2013).

Interestingly, the discovery of MORCs in plants was due to their effect on immunity. A screen for Arabidopsis mutants compromised for recognition to Turnip crinkle virus led to the isolation of CRTI (Kang et al. 2008). Sequence analysis revealed that CRT1 contains the MORC-associated GHKL ATPase and S5 fold domains; thus, it was renamed AtMORC1 (Kang et al. 2010; Moissiard et al. 2012). Further studies in Arabidopsis showed that silencing of AtMORC1 and its closely related homolog, AtMORC2, compromised multiple levels of disease resistance, including nonhost resistance, basal resistance, microbe-associated molecular pattern (MAMP)-triggered immunity, and resistance genemediated immunity (also called effector-triggered immunity) (Kang et al. 2010, 2008, 2012). Silencing MORC1 expression in potato (StMORC1) also negatively affected immunity (Manosalva et al. 2015). By contrast, silencing MORC1 in the closely related plant species tomato (SIMORC1) and Nicotiana benthamiana (NbMORC1) or in the monocot barley (HvMORC1) enhanced disease resistance (Langen et al. 2014; Manosalva et al. 2015). Analyses of immune responses in $N$. benthamiana transiently expressing tomato and potato MORC1s indicated that their ability to suppress or promote immunity, respectively, is controlled by the MORC1 proteins themselves rather than the cellular environment in which they function. Furthermore, domain swapping analyses between these two closely related MORC1s revealed that four amino acid residues located in the carboxyl-terminal region, which contains a linker region downstream of the S5 fold and the C-terminal coil-coiled (CC) domain, regulate this species specificity (Manosalva et al. 2015).

A growing body of evidence suggests that MORCs are critical regulators of epigenetic gene silencing. In Arabidopsis, the expression of transposable elements (TEs) and silenced genes are derepressed in plants carrying mutations in AtMORCl or AtMORC6 (also called DMS11, for defective for meristem silencing) (Brabbs et al. 2013; Lorković 2012; Moissiard et al. 2012). Whether AtMORC1 and AtMORC6 mediate gene silencing by modulating RNA-dependent DNA methylation (Brabbs et al. 2013; Harris et al. 2016; Jing et al. 2016; Lorković 2012; Liu et al. 2016, 2014) or by influencing chromatin structure (Moissiard et al. 2012) is currently unclear. In addition, AtMORC4 and AtMORC7 were shown to suppress nonmethylated genes enriched for those involved in response to pathogen attack (Harris et al. 2016). A genome-wide 
chromatin accessibility study revealed that AtMORC1 is preferentially associated with TEs, a subset of which may help regulate expression of their proximal defense genes (Bordiya et al. 2016). Similar to plants, derepression of a silenced reporter gene was observed in Caenorhabditis elegans deficient for MORC1, the only MORC homolog present in this worm (Moissiard et al. 2012). Mouse male germ cells deficient for MORC1, the first MORC protein identified, displayed elevated TE expression; this phenomenon correlated with decreased de novo DNA methylation during germ cell development (Pastor et al. 2014). HsMORC2 and SMCHD1, also play important roles in epigenetic gene silencing. HsMORC2 downregulates carbonic anhydrase IX expression in tumor cells by recruiting histone deacetylase 4 to the carbonic anhydrase IX promoter, which, in turn, alters histone acetylation levels (Shao et al. 2010), while SMCHD1 is required for the maintenance of $\mathrm{X}$ chromosome inactivation and hypermethylation of CpG islands (Blewitt et al. 2008).

The GHKL-type ATPase domain found in MORCs is present in a wide variety of prokaryotic and eukaryotic proteins that function in DNA structure rearrangement (gyrase and topoisomerase), heat-shock tolerance (HSP90), signal transduction (histidine kinase), and DNA mismatch repair (MutL) (Dutta and Inouye 2000; Lorković 2012). Recent studies, which demonstrated that AtMORC1 and HvMORC1 bind DNA/RNA and exhibit $\mathrm{Mn}^{2+}$ dependent endonuclease activity (Kang et al. 2012; Langen et al. 2014) and that AtMORC1, AtMORC6, HvMORC1, SIMORC1, and StMORC1 possess ATPase activity (Kang et al. 2010; Langen et al. 2014; Lorković 2012; Lorković et al. 2012; Manosalva et al. 2015), suggest that MORCs also are involved in either DNA recombination and repair, chromatin modification, or both. Consistent with this possibility, the Atmorc1/Atmorc2 double mutant displayed enhanced tolerance to the DNA-damaging agent mitomycin $\mathrm{C}$, suggesting that these proteins have a negative effect on DNA repair (Kang et al. 2012). HsMORC2 also was recently shown to play a key role in DNA damage repair, although it promotes DNA repair in human cells treated with ionizing radiation ( $\mathrm{Li}$ et al. 2012).

To elucidate one or more mechanisms through which MORC proteins impact immunity, epigenetic gene silencing, and DNA modification and repair, we characterized the enzymatic properties of SIMORC1 and compared them with those of StMORC1, HsMORC2, and HsMORC3. Here, we report that plant MORCs display many enzymatic properties similar to those of type II topoisomerases (topo IIs). Topoisomerases are critical enzymes that resolve DNA topology issues that arise during transcription, replication, recombination, and repair in both prokaryotes and eukaryotes (Champoux 2001). Topoisomerases that cleave only one strand of the DNA are defined as type I, while topoisomerases that cleave both strands of DNA to generate a double-strand break (ds) are defined as type II. Analysis of SIMORC1 revealed that it contains a K loop-like sequence that, like the $\mathrm{K}$ loop of topo IIs, is required both for DNA-mediated stimulation of ATPase activity and for efficient DNA relaxation and catenation activities. Plant and animal MORCs, like human topo II $\alpha$ (Bau et al. 2014), bind and are regulated by salicylic acid (SA), a critical regulator of immunity in plants and an important regulator of inflammation in animals. Despite these similarities, MORCs and topo IIs differ in important aspects, including the need for one or more accessory factors present in cell extracts, which are required for its ATPdependent efficient conversion of supercoiled DNA into relaxed/nicked DNA, catenanes, and topoisomer intermediates.

\section{RESULTS}

\section{SIMORC1 relaxes and catenates supercoiled DNA.}

Previously, we reported that Arabidopsis MORC1 exhibits endonuclease activity in vitro (Kang et al. 2012). To investigate the possibility that SIMORC1 from tomato (Solanum lycopersicum) also possesses DNA-modifying activities, we assessed its ability to nick/relax negatively supercoiled plasmid DNA. In the presence of increasing concentrations of SIMORC1, the amount of supercoiled pUC19 DNA decreased while the level of nicked/relaxed DNA increased (Fig. 1A). Interestingly, a band that migrated more slowly than relaxed DNA became evident at higher concentrations of SIMORC1. Since this high-molecular weight band was observed even though the reaction mixture was treated with sodium dodecyl sulfate (SDS) and proteinase $\mathrm{K}$ before gel electrophoresis, it is unlikely to be DNA-SIMORC1 complex. A longer exposure of the gel revealed at least three additional high-molecular weight bands (Fig. 1B); the sizes of these three bands correspond to dimers, trimers, and tetramers of the plasmid, suggesting that they may
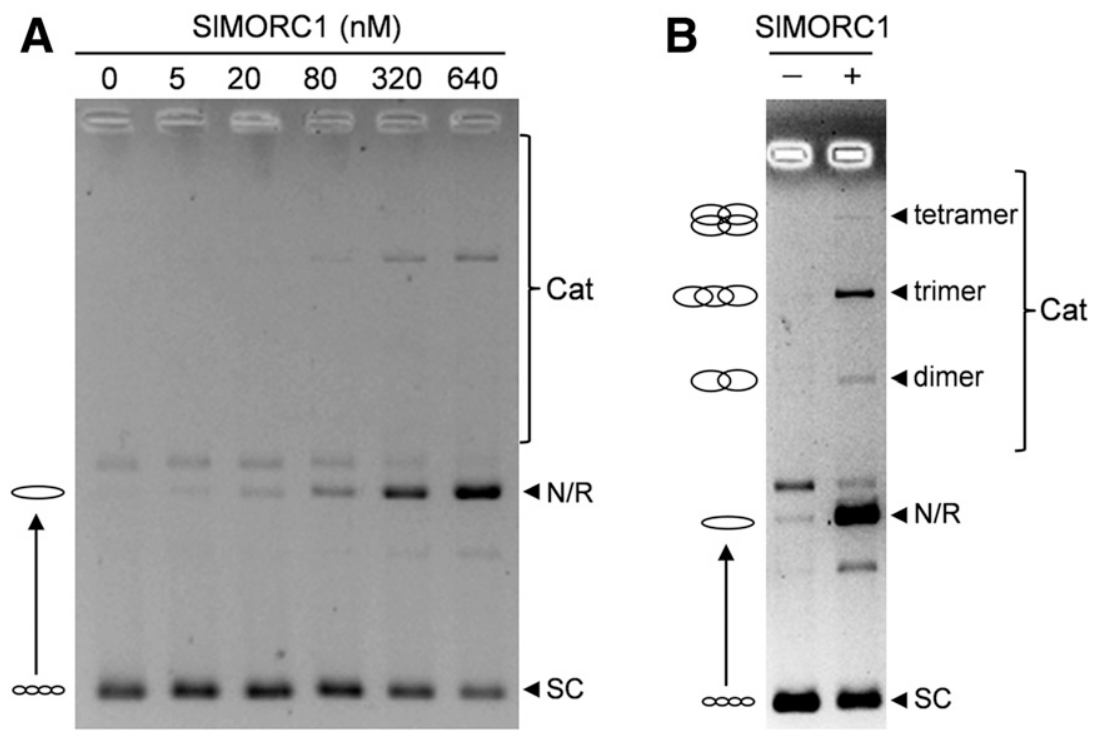

Fig. 1. SIMORC1 relaxes and catenates negatively supercoiled plasmid DNA. A, Concentration-dependent relaxation and catenation of negatively supercoiled pUC19 DNA (7.5 nM) by SIMORC1. Positions of catenated (Cat), nicked/relaxed (N/R), and supercoiled (SC) DNAs are indicated. B, Relaxation and catenation of pUC19 DNA $(7.5 \mathrm{nM})$ by SIMORC1 $(1 \mu \mathrm{M})$. The agarose gel shown in B was exposed for a longer time than that shown in A, to visualize weak bands corresponding to catenane dimers, trimers, and tetramers. 
be interlinked circular DNA called catenanes (Ahumada and Tse-Dinh 2002; Baldi et al. 1980; Tse and Wang 1980). A similar pattern of DNA relaxation and catenation was observed in the presence of increasing concentrations of StMORC1 from potato (Solanum tuberosum) (Supplementary Fig. S1A), indicating that this phenomenon is not limited to SIMORC1. The discovery that SIMORC1 is likely generating catenanes from supercoiled DNA (Fig. 1B) suggested that it has topoisomeraselike activity. Furthermore, since catenation mediated by all members of the topo I subfamily requires the presence of an exposed single-stranded region within the substrate DNA, whereas that mediated by topo II proteins does not (Champoux 2001), SIMORC1's ability to generate catenanes from supercoiled DNA lacking single-stranded gaps suggested that it has topo II-like activity.

\section{SIMORC1 covalently binds dsDNA, has DNA-stimulated ATPase activity, and exhibits topo II-like decatenating activity.}

To establish the catalytic nature of SIMORC1 DNAmodifying activity, several key topo II enzyme assays were employed. A hallmark of topo II-mediated dsDNA cleavage is the formation of a covalent linkage between the enzyme and the
5' ends of both cleaved strands (Champoux 2001; Zechiedrich et al. 1989). To assess whether SIMORC1 forms a covalent bond with DNA, SIMORC1 was incubated with supercoiled plasmid DNA. After terminating the reaction with EDTA and with and without SDS, the reaction was treated with and without proteinase $\mathrm{K}$ to digest away covalently linked as well as free SIMORC1 from DNA before electrophoresis. In the absence of proteinase $\mathrm{K}$, the SIMORC1-treated DNA barely migrated into the gel and was largely retained in the loading well (Fig. 2A, lanes 3 and 4). After proteinase $\mathrm{K}$ treatment, this material was not observed and nicked/relaxed and linear DNA products were instead detected (Fig. 2A, lanes 5 and 6) at the expected sizes, suggesting that the high-molecular weight product that barely entered the gel in lanes 3 and 4 (Fig. 2A) corresponds to a protein-DNA complex. Longer agarose gel electrophoresis was performed to obtain better separation of supercoiled, linear, and nicked/relaxed bands. The observation that SDS treatment alone was insufficient to release the nicked/relaxed and linear DNA species suggests that they were covalently linked to SIMORC1. Furthermore, the ability of SIMORC1 to generate linear DNA argues that it can cleave both strands of dsDNA, which is a characteristic of type II but not type I topoisomerases.

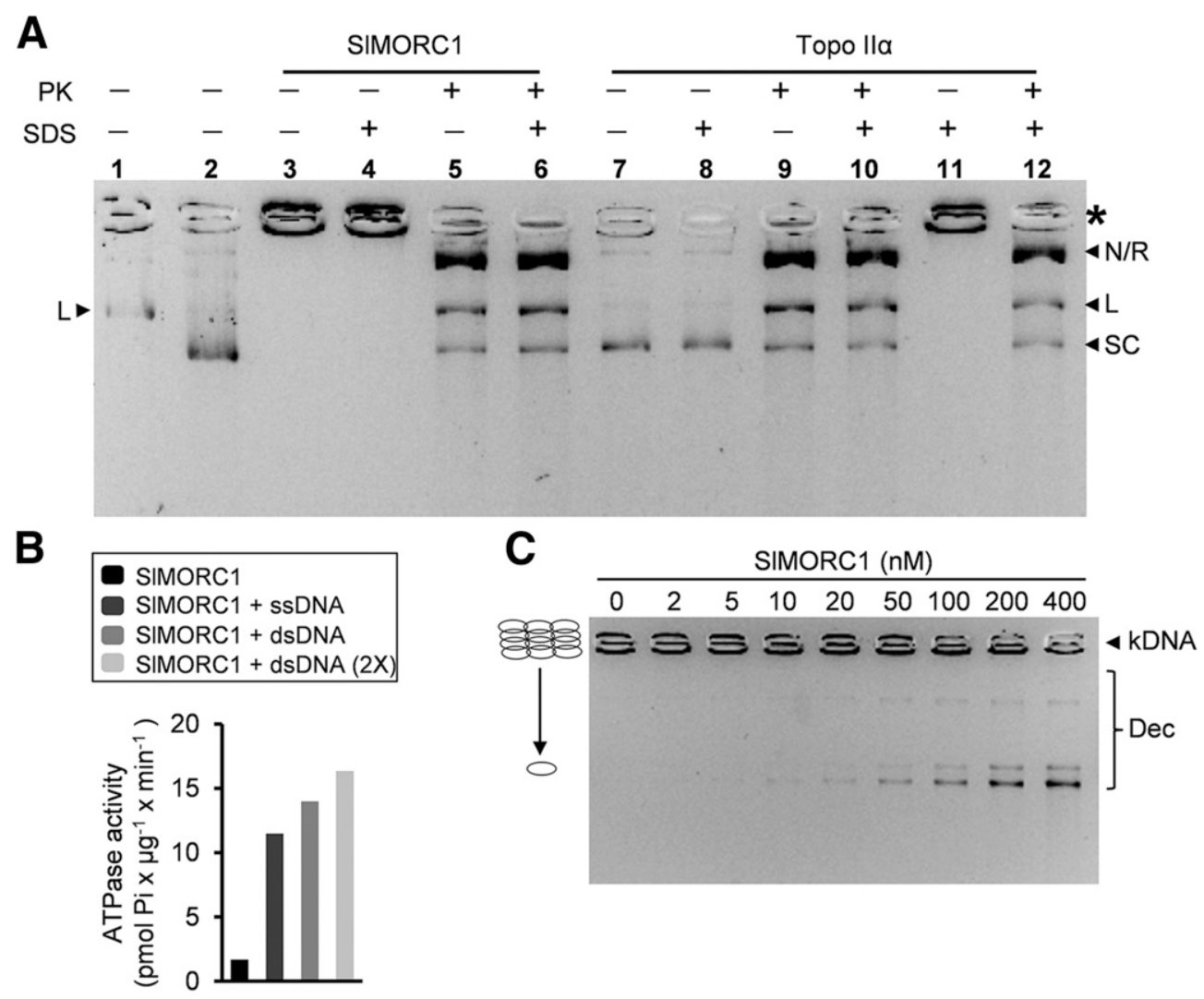

Fig. 2. SIMORC1 exhibits type II topoisomerase (topo II)-like activities. A, SIMOCR1 forms a sodium dodecyl sulfate (SDS)-resistant complex with supercoiled DNA during its conversion to nicked/relaxed and catenane DNA. Negatively supercoiled pER8:HA (hemagglutinin) plasmid DNA (2.5 nM) was incubated with $750 \mathrm{nM}$ SIMORC1 (lanes 3 to 6) or $125 \mathrm{nM}$ of human topoisomerase II $\alpha$ (lanes 7 to 10 in the presence of $\mathrm{Mg}^{2+}$ and lanes 11 and 12 in the presence of $\mathrm{Ca}^{2+}$ in the reaction buffer) for $90 \mathrm{~min}$ at $37^{\circ} \mathrm{C}$. The reactions were terminated by adding stop buffer, which contains EDTA ( \pm SDS) and were then incubated at $37^{\circ} \mathrm{C}$ in the absence (-) or presence (+) of proteinase $\mathrm{K}(\mathrm{PK})$ to remove SIMORC1 from the DNA. Positions of nicked/relaxed (N/R), linear (L), and supercoiled (SC) DNAs are indicated. A linear DNA standard was loaded in lane 1 and untreated DNA plasmid in lane 2. An asterisk indicates the position of the SIMORC1-DNA and topo II $\alpha$-DNA complexes, which, due to their high-molecular weight, cannot migrate through the agarose gel. Notably, SIMORC1DNA complex was stable in the presence of stop buffer containing EDTA $( \pm$ SDS) (lanes 3 and 4 ) but was disrupted by PK treatment (lanes 5 and 6 ), arguing that SIMORC 1 is covalently bound to the DNA. Human topoisomerase II $\alpha$-DNA complex formation activity is enhanced by replacing $\mathrm{Mg}^{2+}$ with $\mathrm{Ca}^{2+}$ in the reaction buffer (compare lanes 7 and 8 to lane 11). B, DNA stimulates the ATPase activity of SIMOCR1. The ATPase activity of SIMORC1 was examined in the absence or presence of different forms of DNA (ssDNA = 6-ng single-strand DNA, dsDNA = 90- or 180-ng double-strand DNA). The data represent the mean of two independent measurements. The experiment was done at least three times with similar results; results from one representative experiment are shown here. C, SIMORC1 decatenates kinetoplast DNA (kDNA, $100 \mathrm{ng}$ ), in a concentration-dependent manner. The positions of kDNA and decatenated (Dec) products are indicated. 
For comparison, commercially available human topoisomerase II $\alpha$ was used as a control to help validate our work with SIMORC1. Note type II topoisomerases exhibit different properties in the presence of $\mathrm{Mg}^{2+}$ and $\mathrm{Ca}^{2+}$. In the absence of ATP and the presence of $\mathrm{Ca}^{2+}$, DNA cleavage can be more efficient than with $\mathrm{Mg}^{2+}$. However with $\mathrm{Ca}^{2+}$, full topoisomerase activity is not found, but, instead, an enzymebound DNA complex results from these reaction conditions (Zechiedrich et al. 1989). Even with $\mathrm{Mg}^{2+}$, the low amount of topo II $\alpha$ used in this assay resulted in only partial substrate processing with some amount of covalent bound product being formed, based on the observation that proteinase $\mathrm{K}$ was required to enable the product to enter the gel (Fig. 2A, compare lanes 7 and 8 with 9 and 10). As expected, when the reaction was performed with $\mathrm{Ca}^{2+}$, covalently bound protein-DNA complexes readily accumulated, which required proteinase $\mathrm{K}$ digestion to enable the reaction products to enter the gel and run with the expected mobility (Fig. 2A, compare lanes 11 and 12). This comparison of the results obtained with SIMORC1 and topo II $\alpha$ are consistent with the idea that SIMORC1 has a topo II-like activity that is unable to mediate a complete reaction cycle, thereby accumulating a covalently linked product. As indicated below, we provided evidence that an accessory factor from the host is needed for full topo II activity beyond the topo II-like activity found with SIMORC1 when it is examined alone.

We previously demonstrated that SIMORC1 exhibits ATPase activity in vitro (Manosalva et al. 2015). Given that topo IIs contain a GHKL ATPase domain whose activity is known to be stimulated by both single-stranded (ss)DNA or dsDNA (Ban et al. 1999; Schmidt et al. 2012), we tested whether DNA stimulates SIMORC1 ATPase activity. The ATPase activity of SIMORC1 was enhanced up to approximately six- or eightfold in the presence of ssDNA or dsDNA, respectively (Fig. 2B). A similar enhancement of ATPase activity was observed with StMORC1, indicating that MORCs have DNA-stimulated ATPase activity much like topo IIs.

In addition to relaxation and catenation, topo IIs can unlink interlocked circular DNAs, a process termed decatenation (Krasnow and Cozzarelli 1982; Lee et al. 2013; Marini et al. 1980). Members of the type I topoisomerases subfamily generate a single-stranded break in the DNA and pass another strand of DNA through the gap, whereas type II topoisomerases generate a staggered ds break through which they pass a region of DNA duplex either within or between DNA molecules. Due to the differences in these mechanisms, the ability to decatenate kinetoplast DNA (kDNA) is a hallmark activity specific to topo IIs (Champoux 2001). Therefore, we tested whether SIMORC1 could decatenate kDNA. Upon incubation of SIMORC1 with $\mathrm{kDNA}$, a dose-dependent conversion of $\mathrm{kDNA}$ to decatenated DNA products was observed, indicating that SIMORC1 has topo II-like decatenation activity (Fig. 2C). To ensure that decatenation activity is not limited to SIMORC1, we also tested MORCs from other plant species, including StMORC1, AtMORC1, and HvMORC1 (Hordeum vulgare, barley). The decatenation activity of StMORC1 was comparable to that of SIMORC1, whereas HvMORC1 and AtMORC1 exhibited a weaker but significant level of decatenation activity.

\section{SIMORC1 possesses a K loop-like stretch of lysine residues that is required for efficient DNA relaxation activity and DNA-mediated stimulation of ATPase activity as well as suppression of INF1-induced cell death.}

Located between the GHKL ATPase and the topoisomeraseprimase domain, eukaryotic topo IIs contain a transducer subdomain that is suggested to be responsible for coupling DNA binding with ATP hydrolysis (Oestergaard et al. 2004; Schmidt et al. 2012). This transducer region interacts with DNA via a stretch of up to six consecutive lysine residues (although most topo IIs contain three) that forms the $\mathrm{K}$ loop. Yeast and human topo IIs containing mutations in the $\mathrm{K}$ loop do not display DNA-mediated stimulation of ATP hydrolysis; they also fail to either relax negatively supercoiled DNA, decatenate kDNA, or both (Schmidt et al. 2012). Although the conservation of amino acid sequences between topo IIs and MORCs is low, their similar activities raised the possibility that MORC proteins contain a $\mathrm{K}$ loop-like region. Multiple sequence alignment of MORCs from Arabidopsis, barley, tobacco (N. benthamiana), potato, and tomato, using the PRALINE program, identified three consecutive lysine residues in the C-terminal CC domain that are conserved among the plant species used for alignment except AtMORC1 and HvMORC1; five of the six proteins also contained a lysine located six residues upstream of this site and another lysine located 12 residues downstream (Fig. 3A). Notably, AtMORC1 and HvMORC1, which display weaker decatenation activity than SIMORC1 and StMORC1, showed less conservation, as they only have two lysine residues in the $\mathrm{K}$ loop-like region; HvMORC1 also lacks the upstream lysine residue.

Previous mutational analyses of yeast and human topo IIs showed that multiple substitutions with alanine (A) for the $\mathrm{K}$ loop lysines $(\mathrm{K})$ substantially impaired DNA relaxation activity, whereas individual substitutions in yeast topo II had a small effect (Schmidt et al. 2012). Swapping negatively charged glutamates (E) for the positively charged lysine led to even greater reductions in yeast and human topo II activity, presumably because glutamates disrupted the electrostatic interaction between the $\mathrm{K}$ loop and DNA. Thus, we generated a SIMORC1 mutant $\left(\right.$ SIMORC1 $\left.1_{\text {KKK-AEE }}\right)$ in which the three consecutive lysine residues were altered (K618A, K619E, K620E). As anticipated, the DNA relaxation and catenation activity of SIMORC $1_{\text {KKK-AEE }}$ was reduced as compared with SIMORC1 (Fig. 3B and C). This reduction was quantified using ImageJ to determine the percent of the supercoiled substrate converted to nicked/relaxed, linear, and catenane products by the mutant versus wild-type SIMORC1 (Supplementary Fig. S2). It should be noted that SIMORC1 and SIMORC $1_{\text {KKK-AEE }}$ were expressed and purified under identical conditions and both proteins showed a similar migration behavior in gel filtration chromatography. The ATPase activity of SIMORC1 $1_{\text {KKK-AEE }}$ also was monitored in the presence and absence of DNA. Similar to the K loop mutants of yeast and human topo II (Schmidt et al. 2012), the basal ATPase level of SIMORC $1_{\mathrm{KKK}-\mathrm{AEE}}$ was approximately threefold higher than that of SIMORC1, but DNA-mediated stimulation of ATPase activity was compromised (Fig. 3D).

Transient expression of SIMORC1 in $N$. benthamiana plants was previously shown to suppress cell death induced by INF1, a MAMP from Phytophthora infestans (Manosalva et al. 2015). This suppressive effect, which is regulated by C-terminal region of SIMORC1, reflects the negative effect SIMORC1 exerts on resistance to $P$. infestans in tomato. Since the putative K loop is located in the C-terminus, we tested whether mutations at this site altered SIMORC1-mediated suppression of this immune response. The role of the putative $\mathrm{K}$ loop was assessed by monitoring cell death in $N$. benthamiana plants transiently expressing estradiol-induced SIMORC1 or SIMORC1 $1_{\mathrm{KKK}-\mathrm{AEE}}$ at 4 days after infiltration (dai) of a second construct that encodes INF1 under the constitutive Cauliflower mosaic virus $35 \mathrm{~S}$ promoter. Approximately $50 \%$ of the $N$. benthamiana coexpressing SIMORC1 $1_{\text {KKK-AEE }}$ and INF1 developed partial necrosis (Fig. 3E and F). By contrast, plants coexpressing SIMORC1 and INF1 were completely necrosis-free at this time, and plants coexpressing an empty vector (EV) along with INF1 predominantly displayed partial to complete necrosis. The expression levels of 
SIMORC1 and SIMORC1 $1_{\mathrm{KKK}-\mathrm{AEE}}$ in $N$. benthamiana were comparable (Supplementary Fig. S3), thereby ruling out the possibility that lower levels of the mutant protein were responsible for weaker suppression of INF1-induced cell death. These results suggest that the K loop-like region of plant MORCs is required not only for efficient DNA relaxation, catenation, and coupling of DNA binding to the stimulation of ATPase activity but also for suppression of INF1-induced cell death.

\section{Biochemical characterization} of SIMORC1's topo II-like catalytic activities.

Previously, $\mathrm{Mn}^{2+}$ and, to a lesser extent, $\mathrm{Mg}^{2+}$ but not $\mathrm{Zn}^{2+}$ were shown to act as cofactors for AtMORC1 and HvMORC1 endonuclease activity (Kang et al. 2012; Langen et al. 2014). In addition to divalent cations, some GHKL ATPase superfamily members have conserved and protein-specific monovalent cation binding sites that appear to be essential for their catalytic activities (Hu et al. 2003). Thus, we tested the requirement of divalent and monovalent cations for SIMORC1's enzymatic activities. In the absence of the addition of cations and after pretreatment with EDTA to remove a wide variety of prebound metal ions, especially those used by topoisomerases, purified recombinant SIMORC1 exhibited no enzymatic activity. Addition of $\mathrm{Mg}^{2+}$ or $\mathrm{Mn}^{2+}$ but not of $\mathrm{K}^{+}, \mathrm{Na}^{+}$, or $\mathrm{Ni}^{2+}$ was required for the ATPase activity (Supplementary Fig. S4A). SIMORC1mediated relaxation and catenation of supercoiled DNA and decatenation of kDNA also required $\mathrm{Mg}^{2+}$ or $\mathrm{Mn}^{2+}$. Since high concentrations of $\mathrm{NaCl}$ reduced the DNA relaxation activity of Drosophila topo II (Zechiedrich et al. 1989) and the ATPase activity of MutL (Hu et al. 2003), their effect on SIMORC1's catalytic activities also was assessed. Increasing concentrations of $\mathrm{NaCl}$ suppressed both SIMORC1 ATPase and DNA relaxation

A

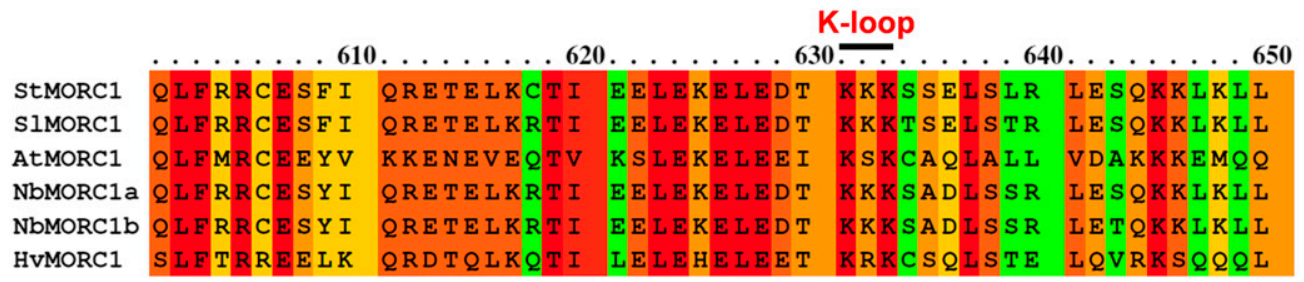

B
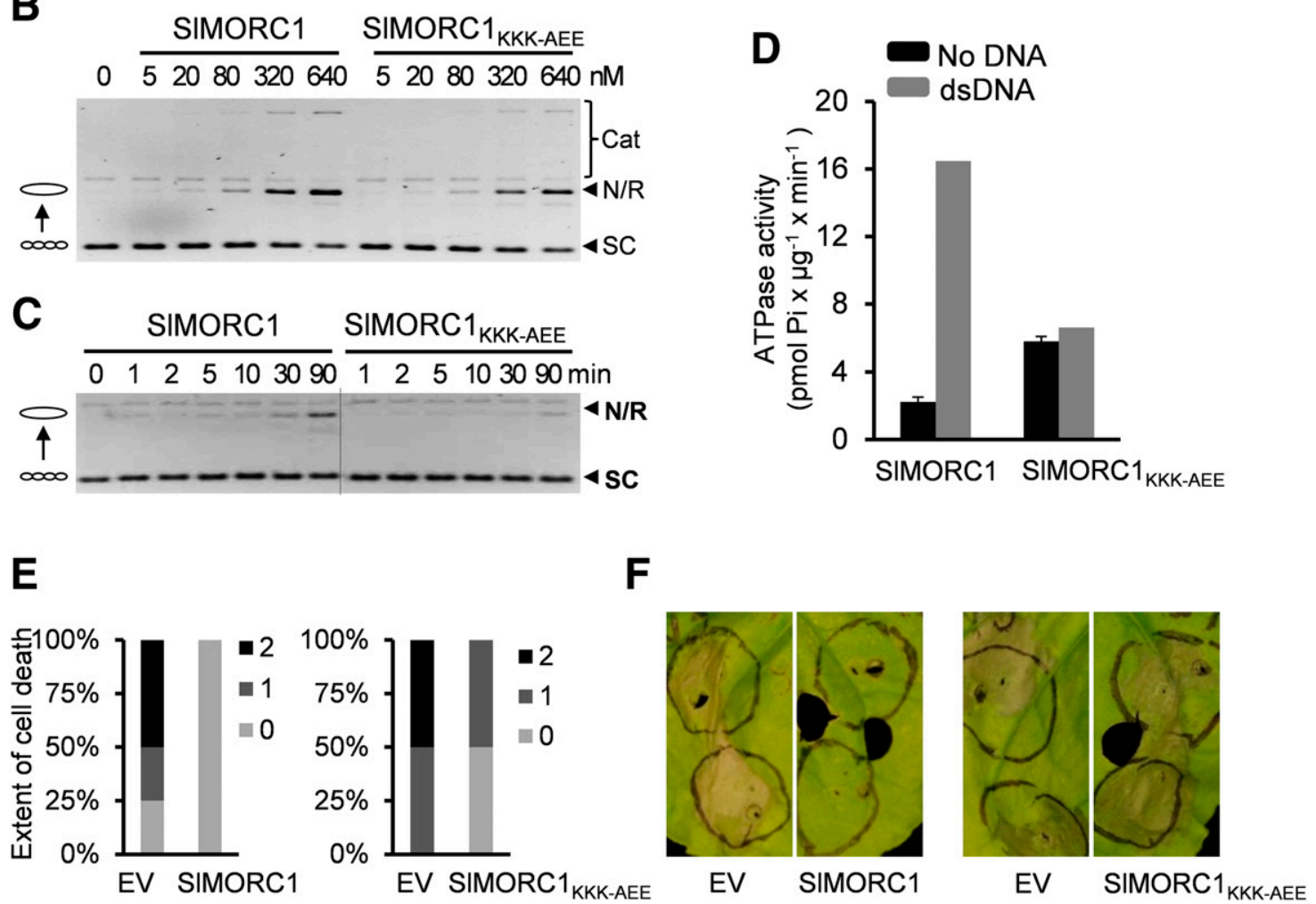

Fig. 3. The putative K loop mutant of SIMORC1 has compromised topoisomerase type II-like activities and reduced ability to suppress INF1-induced cell death A, Amino acid sequence alignment of a segment of MORC1 proteins from different plant species. The putative $\mathrm{K}$ loops are denoted by a black line over the sequences. The color scheme ranges from red, the most conserved alignment position, to green, the least conserved alignment position. $\mathbf{B}$, and $\mathbf{C}$, Concentrationdependent and $\mathbf{C}$, time-dependent relaxation and catenation of negatively supercoiled pUC19 DNA $(7.5 \mathrm{nM})$ by wild-type SIMORC1 and the putative K loop mutant

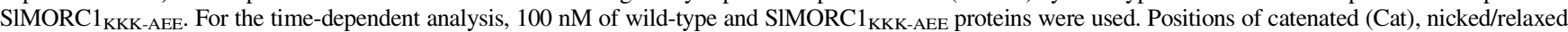
$(\mathrm{N} / \mathrm{R})$, and supercoiled (SC) DNAs are indicated. All lanes shown are from the same gel; a portion of the gel between the SIMORC1 and SIMORC1 $1_{\mathrm{KKK}-\mathrm{AEE}}$ was removed and is indicated by a vertical line. D, SIMORC1 $1_{\mathrm{KKK}-\mathrm{AEE}}$ is defective for DNA-stimulated ATPase activity. The ATPase activity of SIMORC1 and SIMORC1 $1_{\mathrm{KKK}-\mathrm{AEE}}$ was assessed in the presence or absence of double-stranded plasmid DNA $(180 \mathrm{ng})$. The data represent the mean of two independent measurements. The experiment was done at least three times with similar results; results from one representative experiment are shown here. E, Constructs containing N-terminal hemagglutinin-tagged SIMORC1 or SIMORC1 $1_{\text {KKK-AFE }}$ were infiltrated into Nicotiana benthamiana leaves. Transfected leaves were sprayed with $\beta$-estradiol $24 \mathrm{~h}$ after Agrobacterium infiltration to induce protein production. INF1 driven by the constitutive Cauliflower mosaic virus $35 \mathrm{~S}$ promoter was infiltrated $24 \mathrm{~h}$ post $\beta$-estradiol treatment. The extent of INF1-induced cell death was quantified as $0=$ none, $1=$ partial, or $2=$ complete at 4 days after infiltration (dai) of INF1 and is presented graphically. The graphs summarize data obtained from a total of 48 INF1-infitrated areas for each construct; this experiment was done three times with similar results. F, Photographs of $N$. benthamiana leaves were taken 4 dai of INF1. Circles indicate INF1-infiltrated areas on the leaf. 
and catenation activities. Analysis of SIMORC1 DNA relaxation activity under various $\mathrm{pH}$ conditions revealed a broad $\mathrm{pH}$ optimum that spanned $\mathrm{pH} 6.5$ to 8.0. It was previously proposed that acidic $\mathrm{pH}$ shifts the DNA cleavage and religation equilibrium from ds cleavage to single-strand nicking for Drosophila topo II (Zechiedrich et al. 1989). However, this does not appear to be the case with SIMORC1, since the accumulation of relaxed/nicked DNA was significantly reduced at $\mathrm{pH}$ levels below 6.0.

\section{Human MORCs relax and catenate supercoiled DNA and decatenate kDNA.}

Since plant MORCs influence immunity and deregulation of the five human genes encoding MORCs is associated with several human diseases, including cancer ( $\mathrm{Li}$ et al. 2013), we investigated the possibility that human MORCs possess DNAmodifying activities similar to their plant counterparts. Comparison of the amino acid sequence of human versus plant MORCs indicates that there is considerable conservation in the $\mathrm{N}$-terminal portion of the proteins, particularly in the hallmark tripartite GHKL ATPase and S5 fold domains (Supplementary Fig. S5A). In contrast, there is little conservation in the C-terminal portion, which is much longer in human MORCs. Nonetheless, both terminate with a $\mathrm{CC}$ domain. We first assessed the ability of HsMORC2 and HsMORC3 to relax and catenate negatively supercoiled plasmid DNA. In the presence of increasing concentrations of either protein, the amount of supercoiled pUC19 DNA decreased while the level of nicked/relaxed DNA increased (Fig. 4A). Similar to plant MORCs, higher concentrations of HsMORCs also generated high-molecular weight catenanes. HsMORC2 and HsMORC3 also exhibited dose-dependent decatenation activity with kDNA (Fig. 4B), suggesting that human MORCs have topo II-like DNA modification activities comparable to those of plant MORCs. Taken together, these results suggest that the DNA-modifying activities of MORCs are broadly conserved across these two kingdoms.

\section{DNA-modifying activities of MORCs appear to be independent of ATP hydrolysis.}

Topo IIs are known to hydrolyze ATP as part of their catalytic reaction cycle (Bates et al. 2011). Since topo IIs and MORCs contain the GHKL ATPase motif and display DNA-stimulated ATPase activity, we tested whether MORCs also utilize ATP as an energy source to complete their DNA-modifying activities. To assess this possibility, the DNA modification activities of SIMORC1, HsMORC3, and human topo II $\alpha$ were examined in parallel with and without ATP (Fig. 5A). SIMORC1 and HsMORC3 produced similar band patterns, which differed somewhat from those of topo II $\alpha$. Most notably, the DNAmodification activities of topo II $\alpha$ were dramatically increased by the addition of ATP, consistent with previous reports that topo IIs require ATP (Bates et al. 2011; Schmidt et al. 2012). In contrast, added ATP had no effect on the low level of DNA relaxation and catenation activity of SIMORC1 and HsMORC3 in this assay, which contains only buffer, substrate, and purified, recombinant MORCs. Similarly, neither SIMORC1 nor HsMORC3 low levels of decatenation activity were enhanced by ATP, whereas human topo II $\alpha$ efficiently decatenized kDNA in the presence of ATP (Fig. 5B). As a control, we randomly selected mung bean nuclease and tested whether it could decatenate kDNA in our experimental setup. No decatenation was detected, suggesting that the plant and human MORCs were responsible for this, rather than some unspecified nuclease contaminating our MORCs protein preparations (Supplementary Fig. S6). Although these results suggest that MORCs, unlike topo IIs, do not require ATP for their DNA-modifying activities, it is possible that the purified proteins have bound ATP, which enable them to carry out only a portion of the topo II reactions, while multiple reaction cycles require exchange and hydrolysis of added ATP under conditions not recapitulated in our simple in vitro reactions (discussed below).

To further test the apparent ATP independence of the DNAmodifying activities of MORC, we assessed the effect of two GHKL-specific ATPase inhibitors, radicicol and geldanamycin. Consistent with our previous studies, radicicol suppressed the ATPase activity of SIMORC1 (Fig. 5C) (Manosalva et al. 2015); an even greater level of inhibition was observed in the presence of geldanamycin. These results differ from those found with Hsp90, where radicicol binds and inhibits ATPase activity more strongly than geldanamycin (Chène 2002). Neither of these inhibitors altered the DNA relaxation and catenation activity of isolated SIMORC1 (Fig. 5D). The effect of two topo II-specific inhibitors, vp16 (etoposide) and razoxane (Chène 2002), also was tested. Similar to the GHKL-specific ATPase inhibitors, SIMORC1 DNA relaxation and catenation activities were unaffected by the presence of vp16 or razoxane (Fig. 5E). Thus, while MORCs and topo IIs share a set of catalytic domains (ATPase domain and K loop-like region) and exhibit similar enzymatic activities (relaxation, catenation, and decatenation of DNA and covalent binding to DNA), these results suggest that their enzymatic properties and functional mechanisms may be substantially different.

\section{SIMORC1 binds SA, which suppresses} its ATPase and decatenation activities.

Previous studies by Bau and colleagues (Bau and Kurz 2011; Bau et al. 2014) demonstrated that sodium salicylate $(\mathrm{pH}$ neutral SA) specifically suppresses the activities of recombinant human topo II $\alpha$ but not topo II $\beta$, two isoforms of human topo II, or topo I. Mechanistic studies revealed that salicylate primarily inhibits DNA cleavage and secondarily suppresses
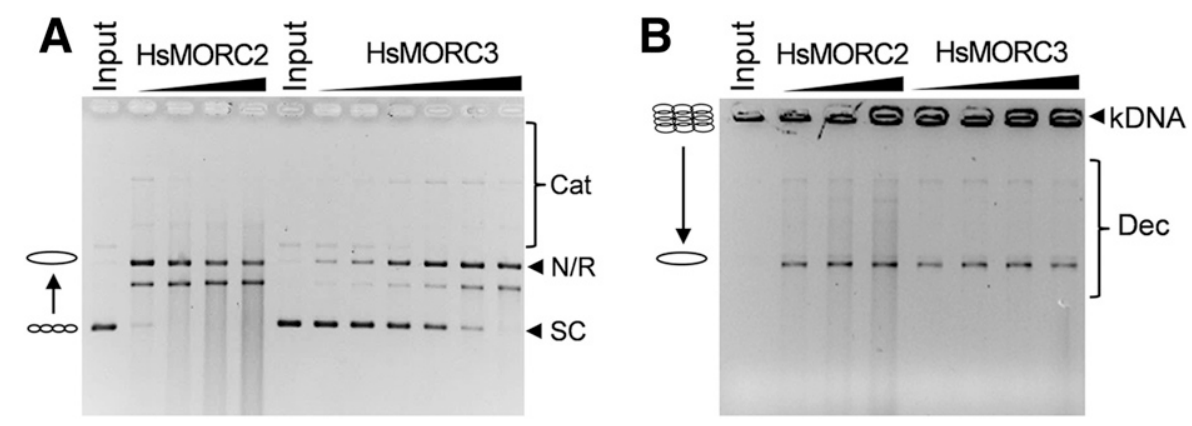

Fig. 4. Human microrchidia (MORC) proteins exhibit topoisomerase type II-like activity. A, Concentration-dependent relaxation and catenation of negatively supercoiled pUC19 DNA (7.5 nM) by HsMORC2 $(25,50,100$, and $200 \mathrm{nM})$ and HsMORC3 $(25,50,100,250,500$, and 1,000 nM). Positions of catenated (Cat), nicked/relaxed (N/R), and supercoiled (SC) DNAs are indicated. B, Concentration-dependent decatenation of kDNA (100 ng) by HsMORC2 (10, 25, and $50 \mathrm{nM})$ and HsMORC3 $(25,50,100$, and $250 \mathrm{nM})$. The positions of kDNA and decatenated (Dec) products are indicated. 
ATPase activity, rather than intercalating into DNA and thereby suppressing the DNA-enzyme interaction or by stabilizing topo II $\alpha$ in a closed clamp configuration on DNA. To assess whether SA affects SIMORC1 activity, we first determined whether SIMORC1 binds to an SA analog, 3-aminoethyl SA (3-AESA), which has been covalently coupled to a surface plasmon resonance (SPR) sensor chip. We previously utilized this method to identify more than two dozen SA-binding proteins from plants and humans (Choi et al. 2015b, 2015a; Manohar et al. 2015; Tian et al. 2012, 2015). Indeed, SIMORC1 showed dose-dependent binding to the 3-AESA-immobilized chip (Fig. 6A). Moreover, increasing concentrations of SA in the binding reaction suppressed SIMORC1 interaction with the 3-AESA immobilized chip, indicating that it represents authentic SA-binding activity (Fig. 6B). In addition, 4hydroxy benzoic acid (4-HBA), which is biologically inactive in plants and is a poor mimic of SA with all tested plants and human SA-binding proteins (Tian et al. 2012), was less effective than SA at competing with 3-AESA for SIMORC1 binding (Fig. 6C). Similar experiments with StMORC1 revealed that it also binds SA (Supplementary Fig. S7). Notably, SA suppressed SIMORC1's ATPase and decatenation activities in a dose-dependent manner (Fig. 6D and E), similar to the reported effects of SA on human topo II $\alpha$ (Bau et al. 2014). However, the DNA relaxation activity of SIMORC1 was unaffected, even at the highest concentration used in the assay (Fig. 6F).

\section{Human MORCs also bind SA, which suppresses their decatenation activity.}

Acetyl SA, commonly known as aspirin, has been widely used as a nonsteroidal anti-inflammatory drug to treat pain and
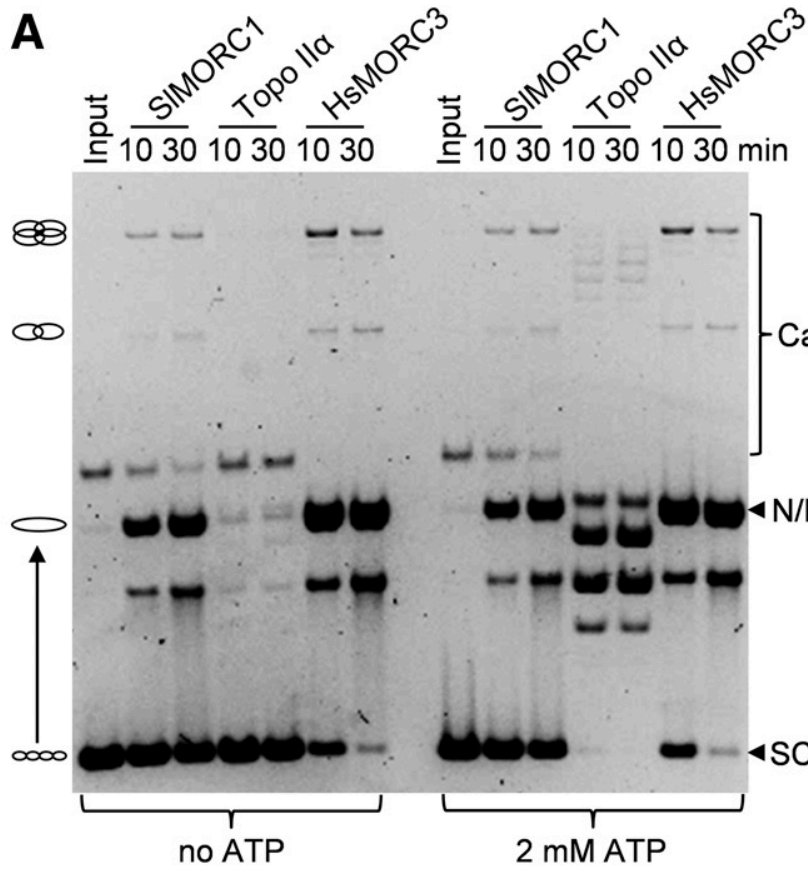

C

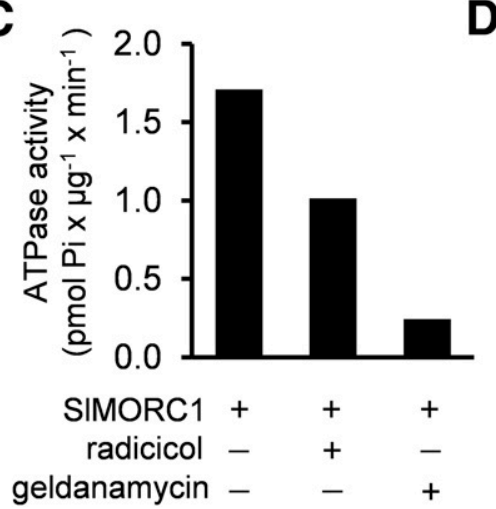

B
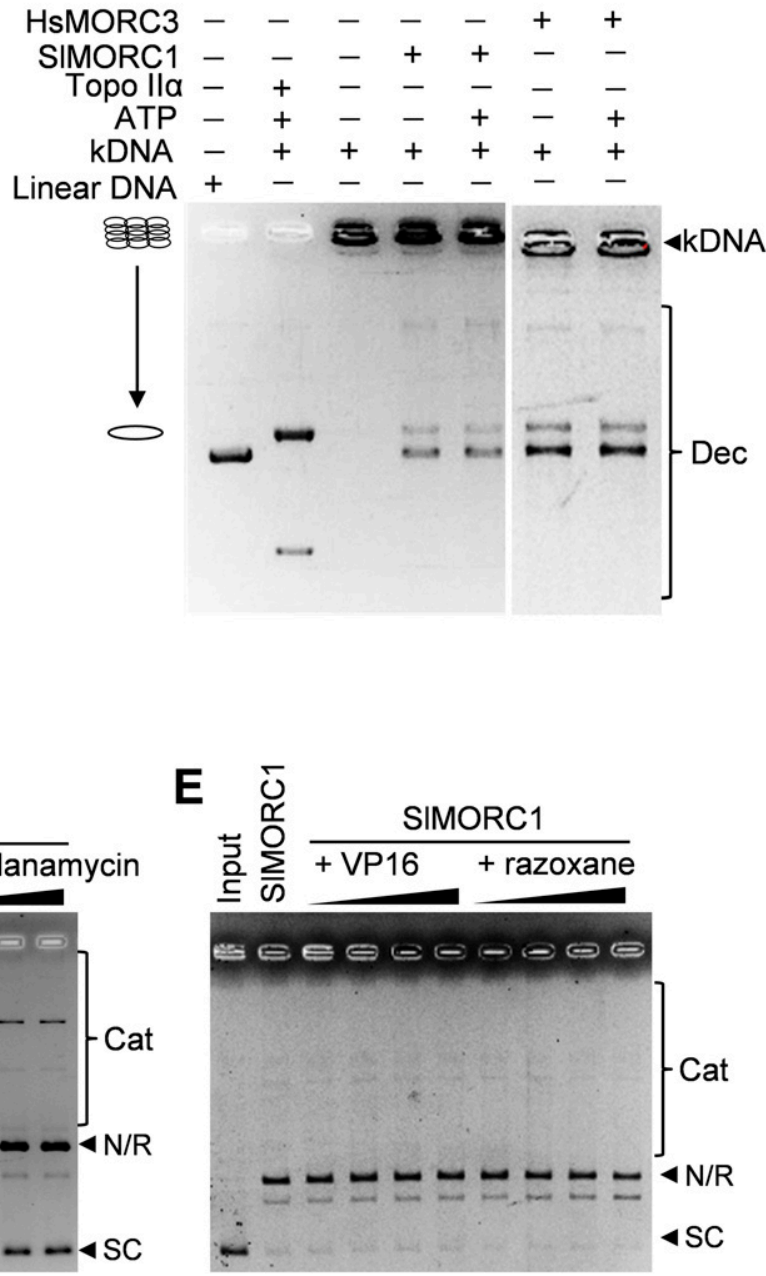

Fig. 5. Plant and human microrchidia (MORC) proteins appear to have distinct DNA-modifying activities compared with topoisomerase type II (topo II). A, DNA relaxation by MORCs (SIMORC1 and HsMORC3) and human topo II $\alpha$. Negatively supercoiled pUC19 DNA (7.5 nM) was incubated with SIMORC1 $(1 \mu \mathrm{M})$, HsMORC3 $(1 \mu \mathrm{M})$, or topo II $\alpha(10$ units) (Topogen Inc.) in the absence or presence of $2 \mathrm{mM}$ ATP for the indicated times. Positions of catenated (Cat), nicked/relaxed (N/R), and supercoiled (SC) DNAs are indicated. B, Decatenation of kDNA (100 ng) by SIMORC1 (500 nM), HsMORC3 (100 nM), or topo II $\alpha$ (10 units) (Topogen Inc.) in the absence or presence of $2 \mathrm{mM}$ ATP. The positions of kDNA and decatenated (Dec) products are indicated. C, The GHKL ATPase-specific inhibitors radicicol and geldanamycin suppress SIMORC1's ATPase activity. The ATPase activity of SIMORC1 was assessed in the absence $(-)$ or presence $(+)$ of $1 \mathrm{mM}$ radicicol or geldanamycin. The data represent the mean of two independent measurements. The ATPase activity assay was done two times with similar results; results from one representative experiment are shown here. D, The GHKL-ATPase-specific inhibitors radicicol and geldanamycin did not affect DNA relaxation and catenation activity of SIMORC1. For the DNA relaxation and catenation assay, SIMORC1 (1 $\mu \mathrm{M})$ was incubated with $7.5 \mathrm{nM}$ pUC19 in the presence of increasing concentrations of radicicol $(0,0.5,1$, and $2 \mathrm{mM})$ or geldanamycin $(0,0.5,1$, and $2 \mathrm{mM})$. E, The topo IIspecific inhibitors VP16 and razoxane did not affect SIMORC1's ability to relax or catenate negatively supercoiled pUC19. SIMORC1 (1 $\mu$ M) was incubated with pUC19 $(5 \mathrm{nM})$ in the presence of increasing concentrations of VP16 or razoxane $(0.1,0.25,0.5$, and $1 \mathrm{mM})$. 
inflammations. However, since aspirin is rapidly metabolized to SA, much of aspirin's bioactivity can be attributed to SA (Choi et al. 2015a, 2015b; Klessig 2016). Identification of targets of aspirin or its primary metabolite, SA, in humans holds great pharmacological promise. This, together with SIMORC1 and StMORC1 demonstrated SA-binding activity, prompted us to test whether HsMORC2 and HsMORC3 also bind SA. Like plant MORCs, HsMORC2 and HsMORC3 bound to the 3-AESAimmobilized chip in a concentration-dependent manner and this binding was partially suppressed by increasing levels of SA (Fig. 7A to D). Moreover, and consistent with their plant counterparts, SA suppressed the decatenation activity of both HsMORC2 and HsMORC3 in a dose-dependent manner (Fig. 7E), but it failed to suppress their DNA relaxation activity (Fig. 7F).

\section{Tomato extracts promote ATP-dependent, efficient} conversion of supercoiled DNA to nicked/relaxed DNA and catenanes and generation of topoisomer intermediates by SIMORC1.

Topoisomerases generate topoisomer intermediates during the conversion of supercoiled DNA to nicked/relaxed DNA, which appear as a ladder of bands migrating between the supercoiled and nicked/relaxed DNA bands on an agarose gel stained with ethidium bromide. The absence of topoisomer intermediates in the reaction products of MORCs, together with the apparent ATP hydrolysis-independent nature of MORC DNA-modifying activities, suggested that these in vitro reactions lacked one or more factors needed for MORCs to carry out multiple cycles of catalysis that required ATP hydrolysis and ATP/ADP exchange. Since one or more such factors should be present in cell extracts, we supplemented the relaxation/catenation reaction with an extract prepared from tomato leaves. Significantly, while, in the SIMORC1-only reaction, $13 \%$ of the supercoiled substrate was converted to products and, in the extract only reaction, 32\% (Fig. 8, lanes 2 and 4), with the SIMORC1-extract combination, essentially all of the supercoiled DNA was converted to product species (97\%) (Fig. 8, lane 5), which was much more than expected for a simple additive effect. To help determine whether the efficient conversion of the supercoiled substrate to products was due to the extract enhancing SIMORC1 topoisomerase activity or the result of SIMORC1 enhancing the low level of topoisomerase activity in the extract or both, we used the SIMORC $1_{\mathrm{KKK} \text {-AEE }}$ mutant. This mutant was found to have reduced DNA relaxation and catenation activity (Fig. 3B and C). This was also evident in Figure 8 , where only $4 \%$ of the supercoiled substrate was converted to products, a value approximating the no-protein control (Fig. 8, lanes 1 and 3). Importantly, while the 52\% conversion by the SIMORC1 $1_{\text {KKK-AEE }}$ mutant-extract combination (lane 6) was slightly more than expected from an additive effect of

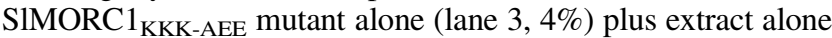
(lane $4,32 \%$ ), it is far below the near total conversion by wildtype protein with extract (lane 5, 97\%), suggesting that the enhanced topoisomerase activity in the combination assay was, at least in part, due to enhancement of SIMORC1 activity by one or more factors in the tomato extract.
A

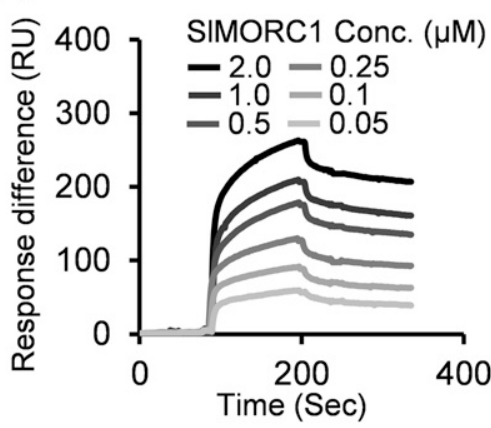

D

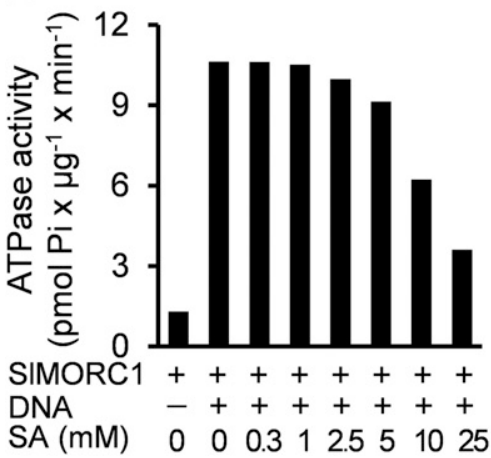

B

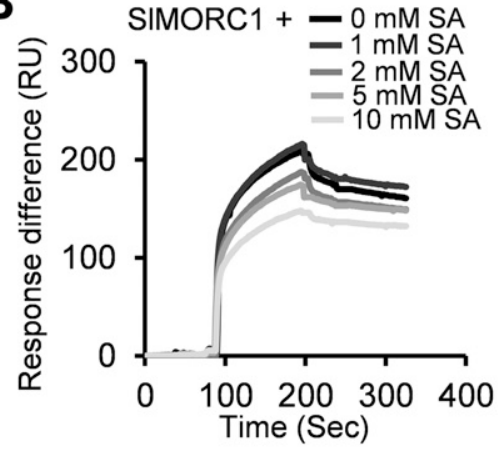

$\mathbf{E}$

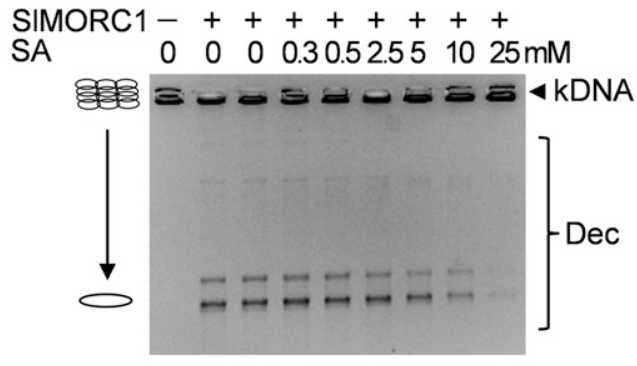

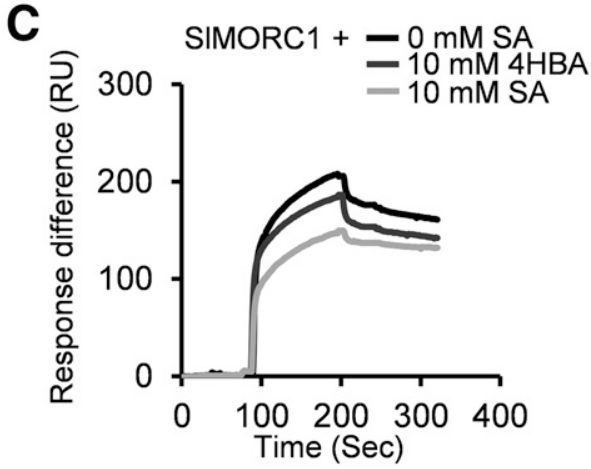

$\mathbf{F}$

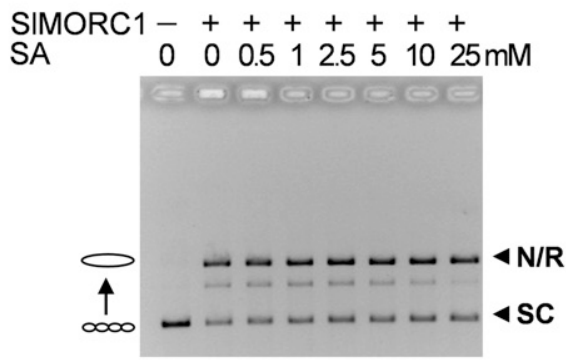

Fig. 6. Salicylic acid (SA) binds SIMORC1 and inhibits its ATPase and decatenation activities but not its relaxation activity. A to C, Surface plasmon resonance (SPR) analyses of SIMORC1's SA-binding activity. A, Sensorgrams obtained with the indicated concentrations of S1MORC1 using a 3-aminoethyl SAimmobilized sensor chip. B, Sensorgrams obtained with SIMORC1 (1 $\mu \mathrm{M})$ in the presence of the indicated concentrations of SA. C, Sensorgrams for SIMORC1 $(1 \mu \mathrm{M})$ in the absence or presence of $10 \mathrm{mM}$ SA or its inactive analog 4-hydroxy benzoic acid (4-HBA). For these SPR analyses, the sensorgram signals detected from a mock-coupled control chip were subtracted (Manohar et al. 2015). D, SA inhibits the DNA-stimulated activation of SIMORC1's ATPase activity. A sample lacking DNA served as the control for basal ATPase activity. Data are the mean of two independent measurements. The experiment was done at least three times with similar results; results from one representative experiment are shown here. E, SA inhibits SIMORC1's decatenation activity. Kinetoplast DNA (kDNA) (100 ng) was incubated with SIMORC1 $(0.2 \mu \mathrm{M})$ in the presence of the indicated concentrations of SA. Positions of kDNA and decatenated (Dec) products are indicated. F, SA does not inhibit the DNA relaxation activity of SIMORC1. Relaxation of negatively supercoiled pUC19 DNA $(7.5 \mathrm{nM})$ by $1 \mu \mathrm{M}$ SIMORC1 in the presence of the indicated concentrations of SA. The reaction was incubated for 10 min instead of 30 min to visualize moderate changes in relaxation of supercoiled DNA. Positions of nicked/relaxed (N/R) and supercoiled (SC) DNAs are indicated. 
We next tested whether the activity of the SIMORC1+ extract is ATP dependent. The GHKL ATPase-specific inhibitor geldanamycin had minimal, if any, effect on SIMORC1 topoisomerase-like activity when the protein was tested without extract (Fig. 8, compare lane 2 and lane 7), as found above (Fig. 5D). However, the inhibitor strongly suppressed the activity in the extract-only reaction, reducing it from $32 \%$ conversion back to background levels (Fig. 8, lanes 1, 4, and 8). The SIMORC1+ extract conversion of supercoiled substrate was also strongly effected by geldanamycin, being reduced from essentially complete conversion of substrate to product (Fig. 8, lane 5) to only $17 \%$ conversion (Fig. 8, lane 9). Consistent with this, addition to the SIMORC1+extract of excess nonhydrolyzable ADP- $\beta-S$ or ATP- $\gamma-\mathrm{S}$ suppressed the DNA processing activity in a dose-dependent manner, conversion of substrate was reduced to 37 to $43 \%$ with $5 \mathrm{mM}$ nonhydrolyzable ADP- $\beta-S$ or ATP- $\gamma-\mathrm{S}$ and 22 to $28 \%$ with $10 \mathrm{mM}$ nonhydrolyzable ADP- $\beta-S$ or ATP- $\gamma$-S (Fig. 8, lanes 5 and 10 to 13). These results suggest that SIMORC1 and likely other plant and human MORCs show a topo II-like activity when tested in isolation but appear to display full topo II activity with the aid of one or more cellular factors. Initial attempts to identify and further characterize one or more enhancing factors in the extract were unsuccessful due to its very unstable nature, even on ice.

\section{DISCUSSION}

In plants, the MORC family of proteins was initially identified and characterized in Arabidopsis and subsequently in tomato, potato, $N$. benthamiana, and barley. These proteins were found to participate in multiple layers of immunity (Kang et al. 2010, 2012; Manosalva et al. 2015; Langen et al. 2014). In addition, AtMORC1, AtMORC2, AtMORC4, AtMORC6, and AtMORC7 were shown to be involved in gene silencing and TE repression (Brabbs et al. 2013; Harris et al. 2016; Lorković et al. 2012; Moissiard et al. 2012, 2014). However, one or more mechanisms by which these proteins regulate plant immunity and gene or TE silencing is presently unclear. In this study, we have characterized the enzymatic and biochemical activity of several purified plant and human MORC proteins and demonstrate that, in isolation, they exhibit topo II-like activity, as they are able to nick or relax and catenate supercoiled DNA molecules and decatenate kDNA. In addition, plant and human MORCs bind SA, which suppresses their ATPase and decatenation activities. Similar to this latter finding, SA suppresses the ATPase and decatenation activities of human topo II $\alpha$ (Bau et al. 2014). However, our analysis also revealed intriguing differences between these proteins. In contrast to topo IIs, the DNA-modifying activities of the MORCs characterized in isolation did not require the addition of ATP nor form topoisomer intermediates in reactions containing only MORCs. Supplementing the SIMORC1-containing reaction with a tomato extract dramatically enhanced conversion of supercoiled DNA to nicked/relaxed DNA and catenanes as well as formation of topoisomer intermediates, all of which were ATP dependent. Since the enhanced activity was reduced when wildtype SIMORC1 was replaced with the K loop-like mutant, one or more factors in the plant extract appear to strongly enhance SIMORC1 activity, including formation of topoisomer intermediates. While, at present, we cannot rule out the possibility that SIMORC1 may be stimulating the endogenous topoisomerase activities in the tomato extract, this model would not explain the extreme lability of the factor found in the extract. Interestingly, while type IIA topoisomerases are large proteins of 750 to $>1,600$ amino acids with all the necessary domains needed to carry out all the reactions being present in a

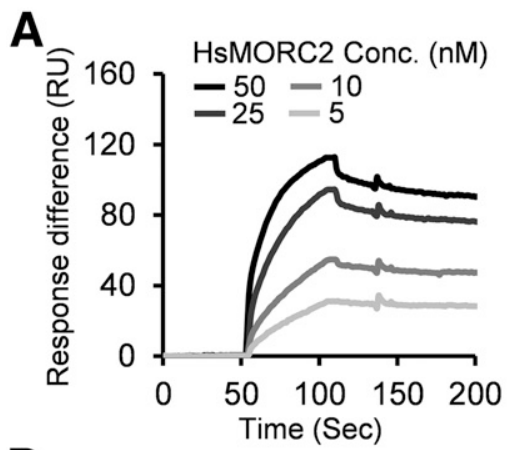

D

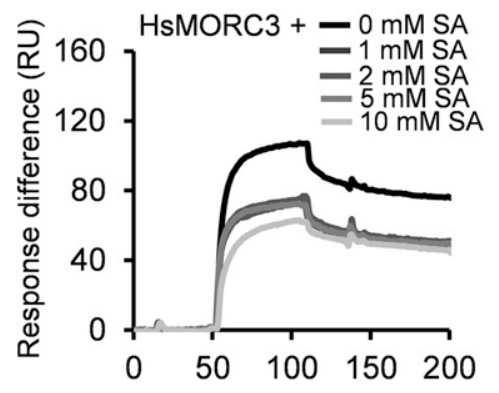

B

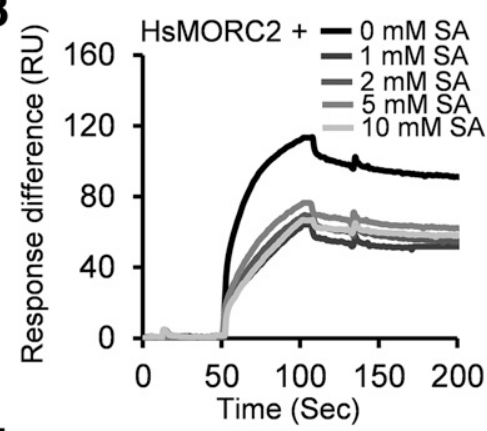

$\mathrm{E}$

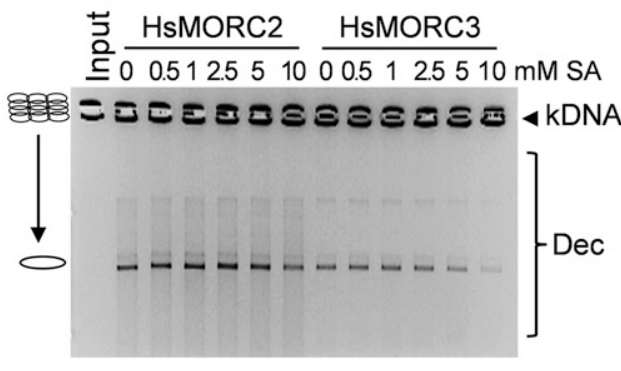

C

$\mathbf{F}$
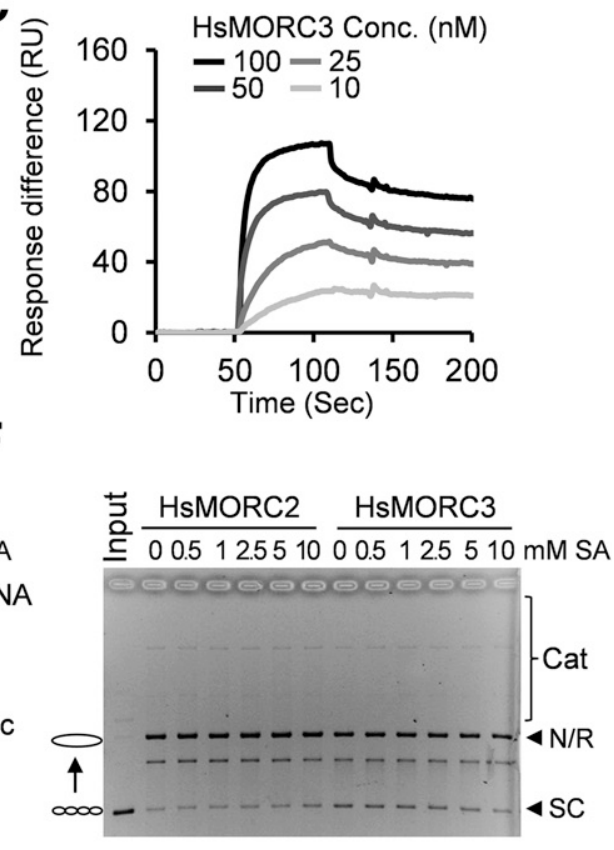

Fig. 7. Salicylic acid (SA) binds human microrchidia (MORC) proteins and inhibits their decatenation activity but not their relaxation activity. A to D, Surface plasmon resonance analyses of the SA-binding activities of HsMORC2 and HsMORC3. Sensorgrams were obtained with the indicated concentrations of HsMORC2 (A) or HsMORC3 (C) using a 3-aminoethyl SA (3-AESA)-immobilized sensor chip or with $50 \mathrm{nM} \mathrm{HsMORC2} \mathrm{(B)} \mathrm{or} 100 \mathrm{nM}$ HsMORC3 (D) in the presence of the indicated concentrations of SA using a 3-AESA-immobilized sensor chip. E, SA inhibits the decatenation activity of human MORCs. Kinetoplast DNA (kDNA) (100 ng) was incubated with $25 \mathrm{nM}$ HsMORC2 or $100 \mathrm{nM}$ HsMORC3 in the presence of the indicated concentrations of SA. Positions of kDNA and decatenated (Dec) products are indicated. F, SA does not inhibit the DNA relaxation activity of human MORCs. Relaxation and catenation of negatively supercoiled pUC19 DNA $(7.5 \mathrm{nM})$ by HsMORC2 (10 nM) or HsMORC3 $(250 \mathrm{nM})$ in the presence of the indicated concentrations of SA. Positions of catenated (Cat), nicked/relaxed (N/R), and supercoiled (SC) DNAs are indicated. 
single molecule, the type IIB topoisomerases found in archaea bacteria consist of two subunits with different domains (Buhler et al. 1998). The N-terminal region of the B subunit shares weak homology with the ATPase domain of the type IIA enzymes and binds ATP. The topo IIB DNA-modifying activities require both subunits $\mathrm{A}$ and $\mathrm{B}$, which form a functional $\mathrm{A}_{2} \mathrm{~B}_{2}$ heterotetramer. In contrast, in isolation, the MORCs cleave and covalently bind to DNA as well as carry out at least one cycle of relaxation, catenation, and decatenation of DNA molecules and require one or more yet-to-be-identified accessory factors for full topo II activity.

Recently the three-dimensional (3-D) structure of the Nterminal segment of the mouse MORC3, including the GHKL ATPase domain, bound to the nucleotide analog AMPPNP was determined ( $\mathrm{Li}$ et al. 2016). The 3-D structure and GHKL ATPase domain-mediated dimerization induction by AMPPNP are consistent with earlier suggestions that the mouse MORC3 may act as a molecular clamp for DNA (Mimura et al. 2010) and the demonstrated trapping of DNA via dimerization through the GHKL ATPase domains of the type II topoisomerases Escherichia coli gyrase and Sulfobus shibatae topoisomerase VI (Champoux 2001; Corbett and Berger 2003). These results are consistent with MORCs functioning as a topo II in vivo.

Enzymes containing GHKL type ATPase domains, including topo IIs, require ATP hydrolysis for their catalytic activities (Dutta and Inouye 2000). DNA gyrase, which was the first topo II characterized, requires ATP hydrolysis in order to introduce negative supercoils into DNA, as this is a thermodynamically unfavorable process. For other topo IIs, ATP hydrolysis has been proposed to enable conformational rearrangements of the enzyme during catalysis rather than to drive DNA modification activities (Bates et al. 2011). Apparently conformational rearrangement and DNA modification activities are interconnected for topo IIs, since neither is accomplished in the absence of ATP. Analysis of human and yeast topo IIs have demonstrated that their ATPase activity is stimulated by DNA binding. This stimulation is mediated by the $\mathrm{K}$ loop, which is a stretch of three or four consecutive lysines in these species (Schmidt et al. 2012). The $\mathrm{K}$ loop is also required for relaxation of supercoiled DNA. Consistent with SIMORC1 topo II-like DNA modification activities, its ATPase activity was stimulated by ssDNA or dsDNA, and a stretch of three lysines located in the C-terminal region (residues 618 to 620) was required both for this ATPase stimulation and for efficient DNA relaxation and catenation activities. These results argue that, despite the difference between the primary amino acid sequences and domains of SIMORC1 and topo IIs, lysine residues 618 to 620 of SIMORC1 function analogously to the K loop of topo IIs. The biological significance of this SIMORC1 K loop-like region was revealed through analyses of $N$. benthamiana plants expressing SIMORC1 $1_{\text {KKK-AEE }}$ (Fig. 3). While SIMORC1 fully suppressed INF1-induced cell death in plants, mutant SIMORC1 $1_{\text {KKK-AEE }}$ only partially blocked cell death. Thus, the coupling between DNA-induced ATPase stimulation and DNA relaxation activity appears to be required for efficient SIMORC1-mediated suppression of immune responses. That suppression of cell death was not fully lost in plants expressing SIMORC $1_{\mathrm{KKK} \text {-AEE }}$ may reflect the fact that this mutant's DNA relaxation and catenation activities were only partially lost.

SIMORC1 displays interesting parallels with human MORCs. Despite their similar enzymatic activities in vitro, however, plant and human MORCs have opposing roles in chromatin condensation. Analysis of atmorcl and atmorc6 mutants in Arabidopsis suggest that AtMORC1 and AtMORC6 are required for chromatin condensation (Moissiard et al. 2012). By contrast, HsMORC2 mediates ATPase-dependent chromatin relaxation in response to radiation-induced ds breaks in DNA

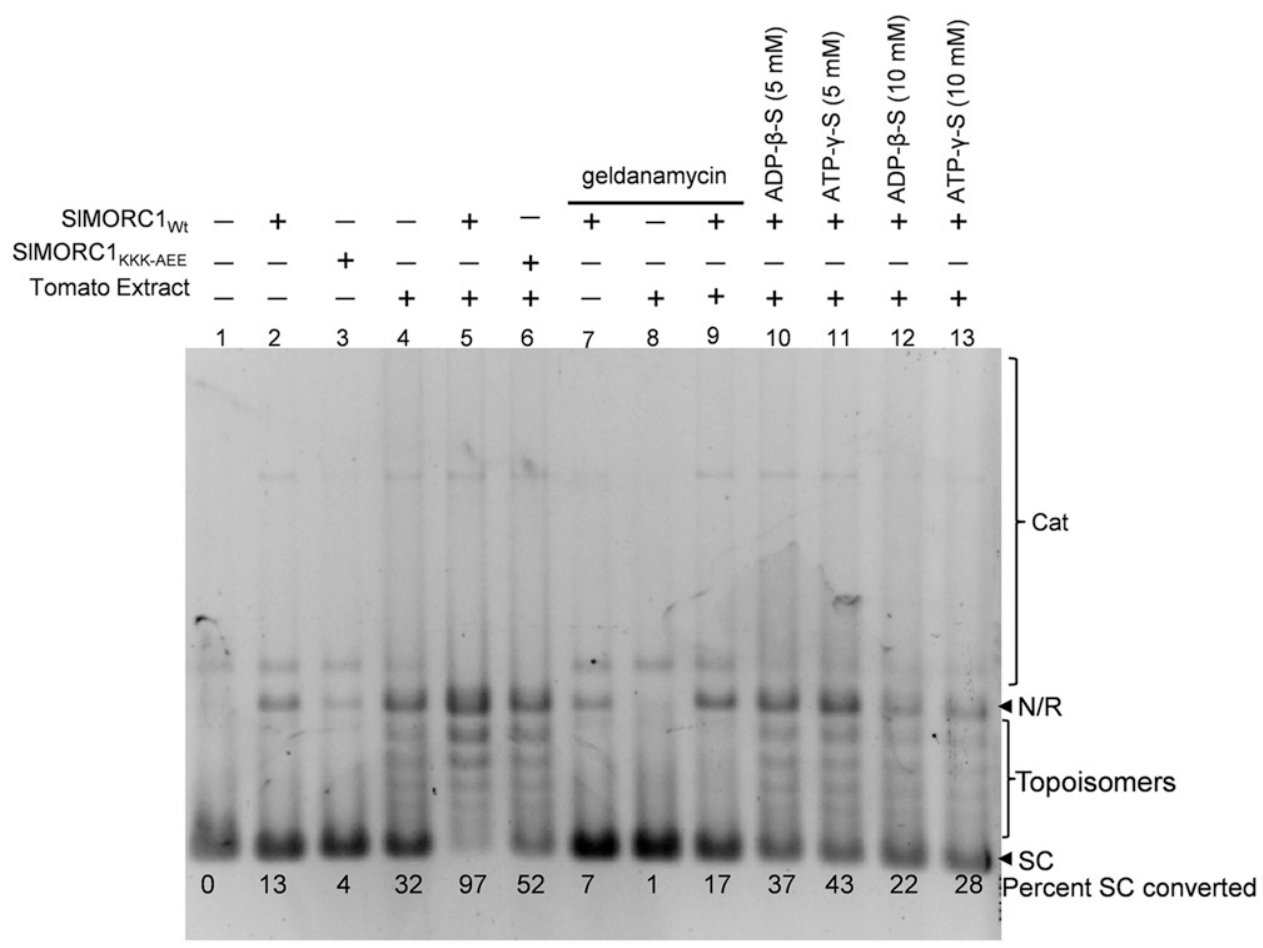

Fig. 8. Tomato extract promotes ATP-dependent relaxation of supercoiled DNA and formation of topoisomer intermediates by SIMORC1. Relaxation and catenation of negatively supercoiled pUC19 DNA $(7.5 \mathrm{nM})$ by $1 \mu \mathrm{M}$ SIMORC1 (lanes 2 and 5) or SIMORC1 $1_{\mathrm{KKK}-\mathrm{AEE}}($ lanes 3 and 6$)$ was assessed in the presence $(+)$ or absence $(-)$ of $10 \mu \mathrm{g}$ of tomato protein extract. The reactions were incubated for 20 min at $30^{\circ} \mathrm{C}$ and were terminated by adding stop buffer, which contains sodium dodecyl sulfate and EDTA and, then, the reactions were incubated at $37^{\circ} \mathrm{C}$ in the presence of proteinase $\mathrm{K}$ (PK). The effects on the enhanced relaxation and catenation activities of the SIMORC1 (1 mM) and extract ( $10 \mathrm{mg})$ combination of $2 \mathrm{mM}$ geldanamycin (lanes 7 to 9$)$ or $5 \mathrm{mM}$ and $10 \mathrm{mM}$ nonhydrolysable ADP or ATP (lanes 10 to 13) are shown. Positions of nicked/relaxed (N/R), supercoiled (SC), catenanes (Cat), and topoisomers are indicated in the gel. The numbers on the bottom of the gel indicate the percentage of supercoiled DNA converted into product. 
(Li et al. 2012). These different effects the proteins exert on condensation are consistent with their divergent functions in DNA damage repair. HsMORC2 promotes DNA damage repair, presumably by increasing the accessibility of DNA lesions to the repair machinery (Li et al. 2012). By contrast, AtMORC1 and its closest homolog, AtMORC2, appear to negatively regulate DNA repair, as the atmorc1 atmorc2 double mutant displays enhanced tolerance to the DNA-damaging agent mitomycin C (Kang et al. 2012).

Several of the Arabidopsis MORCs and HsMORC2 also have been shown to participate in silencing or repression of gene expression. Consistent with the role of AtMORCs in condensing chromatin, mutations in AtMORCl or AtMORC6 resulted in derepression of silenced reporter genes and TEs (Lorković et al. 2012; Moissiard et al. 2012, 2014). Similarly, knocking down expression of HsMORC2 led to a dramatic increase in the expression of CAIX, a protein that plays an important role in tumor cell growth and survival, while overexpression of HsMORC2 suppressed it (Shao et al. 2010). Although the involvement of SIMORC1 topo II-like activities in gene silencing has not been demonstrated, one possibility is the following: epigenetic regulation of gene expression and TE activity requires access to the DNA and its bound histone octamers. Perhaps MORC DNA-modifying activities are required to relieve the topological constraints that develop during epigenetic-associated DNA remodeling, including unwinding of higher order chromatin and even of the DNA duplex (Champoux 2001; Wang 2002).

The demonstration that HsMORC2 inhibits CAIX transcription indirectly, via its interaction with histone deacetylase 4 (Shao et al. 2010), suggests that the function of a given MORC protein can be modulated by its interaction with various partner proteins. Consistent with this possibility, AtMORC1 interacts with more than a dozen different proteins, including other MORCs (D. F. Klessig and H.-G. Kang unpublished data), and AtMORC6 interacts with AtMORC1, AtMORC2, and DMS3 (Lorković et al. 2012; Moissiard et al. 2014). In humans, HsMORC3 recruits the phosphorylated tumor suppressor p53 into a macromolecular structure called promyelocytic leukemia-nuclear body to transduce cancer signaling (Mimura et al. 2010; Takahashi et al. 2007). Additionally, in breast cancer cells, HsMORC2 interacts with and regulate adenosinetriphosphate-citrate lyase, an enzyme essential for lipogenesis (Sánchez-Solana et al. 2014). The GHKL ATPase-specific inhibitors geldanamycin and radicicol bind to the ATP-binding site and inhibit the ATPase activity of HSP90 and histidine kinase (reviewed by Chène [2002]) as well as topo IIs (Chène 2002; Corbett and Berger 2006). Geldanamycin binding to HSP90 prevents its association with various oncoproteins; similar activity was observed with radicicol, which also has antitumor activity (Chène 2002). In light of the finding that HsMORC2 and HsMORC3 bind SA, which suppresses their decatenating activity, it would be interesting to determine whether SA, like other antitumor drugs, can interfere with the ATP binding site of MORCs, thereby altering their interaction with oncoproteins.

We recently demonstrated that SIMORC1 and HvMORC1 negatively affect immune responses in tomato and barley, while AtMORC1 and StMORC1 positively affect immunity in Arabidopsis and potato (Kang et al. 2010, 2012; Langen et al. 2014; Manosalva et al. 2015). Domain swapping analyses of SIMORC1 and StMORC1 revealed that their species-specific effect on immunity is determined by their C-terminal regions rather than differences in the cellular environment in which they function (Manosalva et al. 2015). The results presented in this paper argue that StMORC1, like SIMORC1, is a topo II-like enzyme, as it exhibits DNA-stimulated ATPase activity, relaxes or catenates supercoiled plasmid DNA and decatenates kDNA and likely possesses full topo II activity with one or more accessory factors. Thus, the divergent effects SIMORC1 and StMORC1 exert on immunity do not appear to be due to differences in their DNA modification activities. This finding suggests that MORC topo II activities may represent their evolutionarily conserved core function, upon which are elaborated more recently evolved activities related to immune responses. In addition, the finding that either DNA relaxation, the coupling of DNA binding to the stimulation of ATPase activity, or both is important for SIMORC1-mediated suppression of immune responses argues that SIMORC1 DNA modification activities and its role in immunity are interconnected.

In conclusion, based on the evidence presented here, we propose that MORCs from plants and animals are topo II-like enzymes that differ from type IIA topoisomerases, which catalyze their reactions without the need for one or more accessory factors. In contrast, MORCs require one or more factors present in cell extracts for their efficient DNA-modifying activities. It is possible that they are like archaea DNA topoisomerase VI from Sulfobus shibatae, the prototypic type IIB enzyme, which has two different subunits, both of which are required for most of its topo II activities. It is also possible that a noncanonical topoisomerase-like enzyme or factor works with the MORC proteins to give full topoisomerase activity. Future work based on the information gained from the work presented here should provide insight into how MORC activities are regulated in its multiple roles in plants and humans.

\section{MATERIALS AND METHODS}

\section{Plant materials.}

Seeds of Nicotiana benthamiana plants were germinated in trays and were grown under $16 \mathrm{~h}$ of light and $8 \mathrm{~h}$ of dark, $65 \%$ humidity, and a temperature of $24^{\circ} \mathrm{C}$ during daylight and $22^{\circ} \mathrm{C}$ at night. Plants were transferred to large pots 2 weeks postgermination.

\section{Sequence analyses.}

Multiple sequence analyses of the plant MORCs for the alignment of $\mathrm{K}$ loop were performed using Praline multiple sequence alignment software and Boxshade. Multiple sequence analyses of plant and human MORCs were performed using MultiAlin software.

\section{Cloning and plasmid constructs.}

Cloning of SIMORC1 and StMORC1 were described previously (Manosalva et al. 2015). Site-directed mutagenesis of SIMORC1 to generate SIMORC1 $1_{\mathrm{KKK}-\mathrm{AEE}}$ was performed by wholeplasmid amplification, as described in (Zheng et al. 2004), using primers $\mathrm{F}$, 5' GCAGAGGAAACTTCCGAGCTTTCGACGCR 3' and R, 5'-TTTCCTCTGCAGTGTCCTCTAACTCTTTCTC-3'. HsMORC 2 was amplified by using primers $\mathrm{F}, 5^{\prime}$-GAATTC CAT ATG ATGCTTTGCTTTTTGGATGATG-3' and R, 5'-GAATTC CTCGAG TCAGTCCCCCTTGGTGATGAGG-3' and cDNAs were digested with $\mathrm{Nde} \mathrm{I}$ and $\mathrm{XhoI}$ and were cloned into protein expression vector pET28a (EMD Millipore).

\section{Agrobacterium-mediated transient expression in Nicotiana benthamiana.}

Agrobacterium tumefaciens GV2260 was transformed with pER8:HA (hemagglutinin) plasmids containing EV, SIMORC1, or SIMORC1 mutants expressed under control of a $\beta$-estradiolinducible promoter using electroporation as described previously (Manosalva et al. 2015). Briefly, positive transformants were grown on Luria Bertani (LB) medium, were scraped from plates using a sterile spatula, and were resuspended into sterile infiltration medium (10 mM $\mathrm{MgCl}_{2}, 10 \mathrm{mM}$ morpholineethanesulfonic acid, $\mathrm{pH} 5.5,200 \mu \mathrm{M}$ acetosyringone) and the optical density at $600 \mathrm{~nm}\left(\mathrm{OD}_{600}\right)$ was determined. The suspension was 
diluted in infiltration media to the desired $\mathrm{OD}_{600}$ and was then incubated at room temperature for 1.5 to $2 \mathrm{~h}$. Leaves of 5-weekold $N$. benthamiana plants were infiltrated with agrobacteria carrying EV, SlMORC1, or its mutants at $\mathrm{OD}_{600}=0.7$. Expression was induced 1 dai by spraying plants with a solution containing $30 \mu \mathrm{M} \beta$-estradiol and $0.01 \%$ Tween-20. For INF1-induced cell death, small areas within the EV and MORC1-infiltrated leaves were reinfiltrated with $A$. tumefaciens GV3101 carrying $\mathrm{INF} 1$ driven by the constitutive $35 \mathrm{~S}$ promoter at an $\mathrm{OD}_{600}=0.1$. Cell death was scored between 3 and 5 dai with GV3101.

\section{Protein purifications.}

Three-step protein purification was performed as described previously (Manosalva et al. 2015). Briefly, the Rosetta 2 (DE3) (EMD Millipore) bacterial cells were grown at $37^{\circ} \mathrm{C}$ in LB medium containing $50 \mu \mathrm{g}$ of kanamycin per milliliter and $34 \mu \mathrm{g}$ of chloramphenicol per milliliter until the $\mathrm{OD}_{600}$ of culture reached approximately 0.6, before adding isopropyl$\beta$-D-thiogalactoside to a final concentration of $1 \mathrm{mM}$, to induce gene expression. Induced culture was incubated overnight at $20^{\circ} \mathrm{C}$. The cells were then harvested by centrifugation and the pellet was resuspended into the lysis buffer $(50 \mathrm{mM}$ Tris, $\mathrm{pH}$ $8.5,500 \mathrm{mM} \mathrm{NaCl}, 10 \%$ glycerol, $20 \mathrm{mM}$ imidazole, $0.5 \%$ Triton X-100, and $1 \mathrm{mM}$ phenylmethyl sulphonyl fluoride) and was disrupted by sonication. The clarified supernatant obtained after centrifugation was incubated with Ni-NTA His resin (Novagen) and the bound protein was eluted in lysis buffer supplemented with $250 \mathrm{mM}$ imidazole. The eluted proteins were then subjected to gel filtration chromatography on a HiLoad 16/600 Superdex 200 prep grade column (GE Healthcare) and anion exchange chromatography on a SOURCE 15Q 4.6/100 PE column (GE Healthcare). Human MORC2 and MORC3 was coexpressed with pG-Tf2 plasmid (Takara Bio Inc.) in BL21 (DE3) to improve protein solubility and two step Ni-NTA and gel filtration protein purification was performed as described above.

\section{ATPase assay.}

The ATPase assay was performed using a colorimetric kit (Innova Bioscience) in $50 \mathrm{mM}$ Tris $\mathrm{HCl}, \mathrm{pH} 7.5,2.5 \mathrm{mM}$ $\mathrm{MgCl}_{2}, 10 \mathrm{mM} \mathrm{KCl}$, and $10 \mathrm{mM} \mathrm{NaCl}$ supplemented with $0.2 \mathrm{mM}$ ATP in the absence or presence of ssDNA, dsDNA, geldanamycin, radicicol, $\mathrm{SA}$, or additional $\mathrm{NaCl}$. Reaction mixtures were incubated at $30^{\circ} \mathrm{C}$. ATPase activity was calculated by using a standard curve obtained with a serial dilution of free inorganic phosphate $(\mathrm{Pi})$, and the final plot was adjusted for protein concentration and time of incubation. The specific amount of Pi released, which corresponds to the ATPase activity, was measured and was shown as pmol Pi per microgram of protein per minute. $\mathrm{NaOH}$-neutralized SA of different concentrations was used for suppression of ATPase activities. Commercially available geldanamycin and radicicol (Sigma Aldrich) were used in ATPase activity inhibition assays. All the experiments were done at least twice with similar results unless otherwise specified.

\section{DNA relaxation and decatenation assays.}

DNA relaxation of negatively supercoiled DNA was performed as described by Schmidt et al. (2012), with some modifications. Unless otherwise stated, purified proteins were incubated with $7.5 \mathrm{nM}$ of negatively supercoiled pUC19 or $2.5 \mathrm{nM}$ of pER8:HA plasmid DNA in $20-\mu \mathrm{l}$ reactions for $30 \mathrm{~min}$ at $30^{\circ} \mathrm{C}$. Unless otherwise stated, the final reaction condition included $10 \mathrm{mM}$ Tris, pH 7.5, $100 \mathrm{mM} \mathrm{KCl,} 5 \mathrm{mM} \mathrm{MgCl}_{2}, 2 \%$ glycerol, and $2 \mathrm{mM}$ ATP. Reactions were stopped by the addition of stop buffer containing $0.5 \%$ SDS and $12.5 \mathrm{mM}$ EDTA (final concentration) and were then treated with proteinase $\mathrm{K}$ for $90 \mathrm{~min}$ at $37^{\circ} \mathrm{C}$. Glycerol-based loading dye was added to the samples, which were then seperated on a $1 \%$ agarose gel made with $1 \times$ Tris-acetate-EDTA buffer $(40 \mathrm{mM}$ Tris, $\mathrm{pH} 7.6$, $20 \mathrm{mM}$ acetic acid, and $1 \mathrm{mM}$ EDTA) at 1 to $2 \mathrm{~V} \mathrm{~cm}^{-1}$ for 12 to $16 \mathrm{~h}$. Gels were then stained with $1 \mu \mathrm{g}$ of ethidium bromide per milliliter for 30 to $60 \mathrm{~min}$, followed by destaining for 30 to $60 \mathrm{~min}$. Bands were visualized by UV transillumination. Decatenation assays were performed, using manufacturer guidelines, using human topoisomerase II assay kit (Topogen Inc.). Unless otherwise stated, the reaction was performed in a $20-\mu \mathrm{l}$ volume and was incubated at $37^{\circ} \mathrm{C}$ for $30 \mathrm{~min}$. $\mathrm{NaOH}$-neutralized SA of different concentrations was used in the suppression of relaxation and decatenation activities. Commercially available vp16 (etoposide; Topogen Inc.), razoxane, geldanamycin, and radicicol, ADP- $\beta-S$, ATP- $\gamma-S$ (Sigma Aldrich) were used in DNA relaxation inhibition assays. All the experiments were done at least twice, with similar results unless otherwise specified. Conversion rates for supercoiled DNA in the reactions were calculated following gel analysis with imageJ. Band intensities across the entire lane were collected and corrected for background. The area under each peak value in the graph was collected, except for a band that ran above the nicked/relaxed band that was also found in the no protein control lane. Results are presented as the percent of supercoiled substrate remaining after the reaction as a function of total substrate. Comparing reactions done on different days showed good consistency with standard deviations in the 2 to $4 \%$ range. Tomato protein extract was prepared using frozen 3-weekold tomato leaves, as described previously for Nicotiana benthamiana (Manosalva et al. 2015).

\section{Assessment of 3AESA-binding activities of MORCs by SPR.}

SPR analyses of 3AESA binding and competition by SA or 4-HBA were performed, with a Biacore 3000 instrument (GE Healthcare), as described previously (Manohar et al. 2015). Immobilization of 3AESA on the CM5 sensor chip was performed as described previously (Tian et al. 2012). The specific binding signal was determined by subtracting the signal generated with the control flow cell from the signal generated by the 3AESA-immobilized cell. All the experiments were done at least twice with similar results, unless otherwise specified.

\section{ACKNOWLEDGMENTS}

We thank D. Dempsey for editorial assistance in preparation of the manuscript and E. Richards for insightful discussions. We thank L. Tong (Columbia University) for the Escherichia coli expression vector corresponding to HsMORC3, M. Tian (University of Hawaii) for the INF1 construct, and A. Kumari for assistance in growing plants and green house support. This work was supported by United States National Science Foundation grants IOS 0820405 to D. F. Klessig and MCB-1244227 to J. E. Peters. M. Manohar and D. F. Klessig conceived the research. M. Manohar, C. A. Austin, J. E. Peters, and D. F. Klessig designed the experiments. M. Manohar performed the experiments. M. Manohar, H. W. Choi, P. Manosalva, C. A. Austin, J. E. Peters, and D. F. Klessig analyzed the data. M. Manohar and D. F. Klessig wrote the paper. H. W. Choi, C. A. Austin, and J. E. Peters edited the manuscript. All authors have read and approved the final manuscript.

\section{LITERATURE CITED}

Ahumada, A., and Tse-Dinh, Y.-C. 2002. The role of the $\mathrm{Zn}$ (II) binding domain in the mechanism of E. coli DNA topoisomerase I. BMC Biochem. 3:13.

Baldi, M. I., Benedetti, P., Mattoccia, E., and Tocchini-Valentini, G. P. 1980. In vitro catenation and decatenation of DNA and a novel eucaryotic ATP-dependent topoisomerase. Cell 20:461-467.

Ban, C., Junop, M., and Yang, W. 1999. Transformation of MutL by ATP binding and hydrolysis: A switch in DNA mismatch repair. Cell 97: 85-97. 
Bates, A. D., Berger, J. M., and Maxwell, A. 2011. The ancestral role of ATP hydrolysis in type II topoisomerases: Prevention of DNA doublestrand breaks. Nucleic Acids Res. 39:6327-6339.

Bau, J. T., Kang, Z., Austin, C. A., and Kurz, E. U. 2014. Salicylate, a catalytic inhibitor of topoisomerase II, inhibits DNA cleavage and is selective for the $\alpha$ isoform. Mol. Pharmacol. 85:198-207.

Bau, J. T., and Kurz, E. U. 2011. Sodium salicylate is a novel catalytic inhibitor of human DNA topoisomerase II alpha. Biochem. Pharmacol. $81: 345-354$

Blewitt, M. E., Gendrel, A.-V., Pang, Z., Sparrow, D. B., Whitelaw, N., Craig, J. M., Apedaile, A., Hilton, D. J., Dunwoodie, S. L., Brockdorff, N., Kay, G. F., and Whitelaw, E. 2008. SmcHD1, containing a structuralmaintenance-of-chromosomes hinge domain, has a critical role in $\mathrm{X}$ inactivation. Nat. Genet. 40:663-669.

Bordiya, Y., Yi, Z., Nam, J.-C., Bonnard, A. C., Choi, H. W., Lee B.-K., Kim, J., Klessig, D. F., Fei, Z., and Kang, H.-G. 2016. Pathogen infection and MORC proteins affect chromatin accessibility of transposable elements and expression of their proximal genes in Arabidopsis. Mol. Plant-Microbe Interact. 29:674-687.

Brabbs, T. R., He, Z., Hogg, K., Kamenski, A., Li, Y., Paszkiewicz, K. H., Moore, K. A., O’Toole, P., Graham, I. A., and Jones, L. 2013. The stochastic silencing phenotype of Arabidopsis morc6 mutants reveals a role in efficient RNA-directed DNA methylation. Plant J. 75:836-846.

Buhler, C., Gadelle, D., Forterre, P., Wang, J. C., and Bergerat, A. 1998. Reconstitution of DNA topoisomerase VI of the thermophilic archaeon Sulfolobus shibatae from subunits separately overexpressed in Escherichia coli. Nucleic Acids Res. 26:5157-5162.

Champoux, J. J. 2001. DNA topoisomerases: Structure, function, and mechanism. Annu. Rev. Biochem. 70:369-413.

Chène, P. 2002. ATPases as drug targets: Learning from their structure. Nat. Rev. Drug Discov. 1:665-673.

Choi, H. W., Tian, M., Manohar, M., Harraz, M. M., Park, S.-W., Schroeder, F. C., Snyder, S. H., and Klessig, D. F. 2015a. Human GAPDH is a target of aspirin's primary metabolite salicylic acid and its derivatives. PLoS One 10:e0143447.

Choi, H. W., Tian, M., Song, F., Venereau, E., Preti, A., Park, S.-W. Hamilton, K., Swapna, G. V. T., Manohar, M., Moreau, M., Agresti, A., Gorzanelli, A., De Marchis, F., Wang, H., Antonyak, M., Micikas, R. J., Gentile, D. R., Cerione, R. A., Schroeder, F. C., Montelione, G. T., Bianchi, M. E., and Klessig, D. F. 2015b. Aspirin's active metabolite salicylic acid targets high mobility group box 1 to modulate inflammatory responses. Mol. Med. 21:526-535.

Corbett, K. D., and Berger, J. M. 2003. Emerging roles for plant topoisomerase VI. Chem. Biol. 10:107-111.

Corbett, K. D., and Berger, J. M. 2006. Structural basis for topoisomerase VI inhibition by the anti-Hsp90 drug radicicol. Nucleic Acids Res. 34: 4269-4277.

Dutta, R., and Inouye, M. 2000. GHKL, an emergent ATPase/kinase superfamily. Trends Biochem. Sci. 25:24-28.

Harris, C. J., Husmann, D., Liu, W., Kasmi, F. E., Wang, H., Papikian, A., Pastor, W. A., Moissiard, G., Vashisht, A. A., Dangl, J. L., Wohlschlegel, J. A., and Jacobsen, S. E. 2016. Arabidopsis AtMORC4 and AtMORC7 form nuclear bodies and repress a large number of protein-coding genes. PLoS Genet. 12:e1005998.

Hu, X., Machius, M., and Yang, W. 2003. Monovalent cation dependence and preference of GHKL ATPases and kinases. FEBS Lett. 544:268-273.

Iyer, L. M., Abhiman, S., and Aravind, L. 2008. MutL homologs in restriction-modification systems and the origin of eukaryotic MORC ATPases. Biol. Direct 3:8.

Jing, Y., Sun, H., Yuan, W., Wang, Y., Li, Q., Liu, Y., Li, Y., and Qian, W. 2016. SUVH2 and SUVH9 couple two essential steps for transcriptional gene silencing in Arabidopsis. Mol. Plant 9:1156-1167.

Kang, H.-G., Hyong, W. C., von Einem, S., Manosalva, P., Ehlers, K., Liu, P.-P., Buxa, S. V., Moreau, M., Mang, H.-G., Kachroo, P., Kogel, K. H., and Klessig, D. F. 2012. CRT1 is a nuclear-translocated MORC endonuclease that participates in multiple levels of plant immunity. Nat. Commun. 3:1297.

Kang, H. G., Kuhl, J. C., Kachroo, P., and Klessig, D. F. 2008. CRT1, an Arabidopsis ATPase that interacts with diverse resistance proteins and modulates disease resistance to turnip crinkle virus. Cell Host Microbe 3:48-57.

Kang, H.-G., Oh, C.-S., Sato, M., Katagiri, F., Glazebrook, J., Takahashi, H., Kachroo, P., Martin, G. B., and Klessig, D. F. 2010. Endosomeassociated CRT1 functions early in resistance gene-mediated defense signaling in Arabidopsis and tobacco. Plant Cell 22:918-936.

Klessig, D. F. 2016. Newly identified targets of aspirin and its primary metabolite, salicylic acid. DNA Cell Biol. 35:163-166.
Krasnow, M. A., and Cozzarelli, N. R. 1982. Catenation of DNA rings by topoisomerases. Mechanism of control by spermidine. J. Biol. Chem. 257:2687-2693.

Langen, G., von Einem, S., Koch, A., Imani, J., Pai, S. B., Manohar, M., Ehlers, K., Choi, H. W., Claar, M., Schmidt, R., Mang, H.-G., Bordiya, Y., Kang, H.-G., Klessig, D. F., and Kogel, K.-H. 2014. The compromised recognition of turnip crinkle virus1 subfamily of microrchidia ATPases regulates disease resistance in barley to biotrophic and necrotrophic pathogens. Plant Physiol. 164:866-878.

Lee, I., Dong, K. C., and Berger, J. M. 2013. The role of DNA bending in type IIA topoisomerase function. Nucleic Acids Res. 41:5444-5456.

Li, D. Q., Nair, S. S., and Kumar, R. 2013. The MORC family: New epigenetic regulators of transcription and DNA damage response. Epigenetics 8:685-693.

Li, D. Q., Nair, S. S., Ohshiro, K., Kumar, A., Nair, V. S., Pakala, S. B., Reddy, S. D. N., Gajula, R. P., Eswaran, J., Aravind, L., and Kumar, R. 2012. MORC2 signaling integrates phosphorylation-dependent, ATPasecoupled chromatin remodeling during the DNA damage response. Cell Reports 2:1657-1669.

Li, S., Yen, L., Pastor, W. A., Johnston, J. B., Du, J., Shew, C. J., Liu, W., Ho, J., Stender, B., Clark, A. T., Burlingame, A. L., Daxinger, L., Patel, D. J., and Jacobsen, S. E. 2016. Mouse MORC3 is a GHKL ATPase that localizes to H3K4me3 marked chromatin. Proc. Natl. Acad. Sci. U.S.A. 113. doi:10.1073/pnas. 1609709113

Liu, Z. W., Shao, C. R., Zhang, C. J., Zhou, J. X., Zhang, S. W., Li, L., Chen, S., Huang, H. W., Cai, T., and He, X. J. 2014. The SET domain proteins SUVH2 and SUVH9 are required for Pol V occupancy at RNA-directed DNA methylation loci. PLoS Genet. 10:e1003948.

Liu, Z. W., Zhou, J. X., Huang, H. W., Li, Y. Q., Shao, C. R., Li, L., Cai, T., Chen, S., and He, X. J. 2016. Two components of the RNA-directed DNA methylation pathway associate with MORC6 and silence loci targeted by MORC6 in Arabidopsis. PLoS Genet. 12:e1006026.

Lorković, Z. J. 2012. MORC proteins and epigenetic regulation. Plant Signal. Behav. 7:1561-1565.

Lorković, Z. J., Naumann, U., Matzke, A. J. M., and Matzke, M. 2012. Involvement of a GHKL ATPase in RNA-directed DNA methylation in Arabidopsis thaliana. Curr. Biol. 22:933-938.

Manohar, M., Tian, M., Moreau, M., Park, S.-W., Choi, H. W., Fei, Z., Friso, G., Asif, M., Manosalva, P., von Dahl, C. C., Shi, K., Ma, S., Dinesh-Kumar, S. P., O'Doherty, I., Schroeder, F. C., van Wijk, K. J., and Klessig, D. F. 2015. Identification of multiple salicylic acid-binding proteins using two high throughput screens. Front. Plant Sci. 5:777.

Manosalva, Patricia M; Manohar, Murli; Heinz, Karl-Kogel; Kang, HongGu; Klessig, Daniel, F. 2015. The GHKL ATPase MORC1 modulates species-specific plant immunity in Solanaceae. Mol. Plant-Microbe Interact. 28:927-948.

Marini, J. C., Miller, K. G., and Englund, P. T. 1980. Decatenation of kinetoplast DNA by topoisomerases. J. Biol. Chem. 255:4976-4979.

Mimura, Y., Takahashi, K., Kawata, K., Akazawa, T., and Inoue, N. 2010. Two-step colocalization of MORC3 with PML nuclear bodies. J. Cell Sci. 123:2014-2024.

Moissiard, G., Bischof, S., Husmann, D., Pastor, W. A., Hale, C. J., Yen, L., Stroud, H., Papikian, A., Vashisht, A. A., Wohlschlegel, J. A., and Jacobsen, S. E. 2014. Transcriptional gene silencing by Arabidopsis microrchidia homologues involves the formation of heteromers. Proc. Natl. Acad. Sci. U.S.A. 111:7474-7479.

Moissiard, G., Cokus, S. J., Cary, J., Feng, S., Billi, A. C., Stroud, H., Husmann, D., Zhan, Y., Lajoie, B. R., McCord, R. P., Hale, C. J., Feng, W., Michaels, S. D., Frand, A. R., Pellegrini, M., Dekker, J., Kim, J. K., and Jacobsen, S. E. 2012. MORC family ATPases required for heterochromatin condensation and gene silencing. Science 336:1448-1451.

Oestergaard, V. H., Bjergbaek, L., Skouboe, C., Giangiacomo, L., Knudsen, B. R., and Andersen, A. H. 2004. The transducer domain is important for clamp operation in human DNA topoisomerase IIalpha. J. Biol. Chem. 279:1684-1691.

Pastor, W. A., Stroud, H., Nee, K., Liu, W., Pezic, D., Manakov, S., Lee, S. A., Moissiard, G., Zamudio, N., Bourc'his, D., Aravin, A. A., Clark, A. T., and Jacobsen, S. E. 2014. MORC1 represses transposable elements in the mouse male germline. Nat. Commun. 5:5795.

Sánchez-Solana, B., Li, D. Q., and Kumar, R. 2014. Cytosolic functions of MORC2 in lipogenesis and adipogenesis. CBBA Mol. Cell Res. 1843 316-326.

Schmidt, B. H., Osheroff, N., and Berger, J. M. 2012. Structure of a topoisomerase II-DNA-nucleotide complex reveals a new control mechanism for ATPase activity. Nat. Struct. Mol. Biol. 19:1147-1154.

Shao, Y., Li, Y., Zhang, J., Liu, D., Liu, F., Zhao, Y., Shen, T., and Li, F. 2010. Involvement of histone deacetylation in MORC2-mediated downregulation of carbonic anhydrase IX. Nucleic Acids Res. 38:2813-2824. 
Takahashi, K., Yoshida, N., Murakami, N., Kawata, K., Ishizaki, H., Tanaka-Okamoto, M., Miyoshi, J., Zinn, A. R., Shime, H., and Inoue, N. 2007. Dynamic regulation of p53 subnuclear localization and senescence by MORC3. Mol. Biol. Cell 18:1701-1709.

Tian, M., Sasvari, Z., Gonzalez, P. A., Friso, G., Rowland, E., Liu, X., Wijk, K. J.Van, Nagy, P. D., and Klessig, D. F. 2015. Salicylic acid inhibits the replication of Tomato bushy stunt virus by directly targeting a host component in the replication complex. Mol. Plant-Microbe Interact. 28:379-386.

Tian, M., von Dahl, C. C., Liu, P. P., Friso, G., van Wijk, K. J., and Klessig, D. F. 2012. The combined use of photoaffinity labeling and surface plasmon resonance-based technology identifies multiple salicylic acidbinding proteins. Plant J. 72:1027-1038.

Tse, Y., and Wang, J. C. 1980. E. coli and M. luteus DNA topoisomerase I can catalyze catenation of decatenation of double-stranded DNA rings. Cell 22:269-276.
Wang, J. C. 2002. Cellular roles of DNA topoisomerases: A molecular perspective. Nat. Rev. Mol. Cell Biol. 3:430-440.

Zechiedrich, E. L., Christiansen, K., Andersen, A. H., Westergaard, O., and Osheroff, N. 1989. Double-stranded DNA cleavage/religation reaction of eukaryotic topoisomerase II: Evidence for a nicked DNA intermediate. Biochemistry 28:6229-6236.

Zheng, L., Baumann, U., and Reymond, J.-L. 2004. An efficient one-step sitedirected and site-saturation mutagenesis protocol. Nucleic Acids Res. 32:e115.

\section{AUTHOR-RECOMMENDED INTERNET RESOURCES}

Boxshade server: http://www.ch.embnet.org/software/BOX_form.html MultiAlin software: http://multalin.toulouse.inra.fr/multalin

Praline multiple sequence alignment software: http://www.ibi.vu.nl/programs/pralinewww 